\title{
Spectrum Allocation and Reuse in 5G New Radio on Licensed and Unlicensed Millimeter-Wave Bands in Indoor Environments
}

\author{
Rony Kumer Saha \\ Radio and Spectrum Laboratory KDDI Research, Inc., 2-1-15 Ohara, Fujimino-shi, Saitama, Japan \\ Correspondence should be addressed to Rony Kumer Saha; rony107976@gmail.com
}

Received 13 February 2021; Revised 3 March 2021; Accepted 17 March 2021; Published 5 April 2021

Academic Editor: Ding Xu

Copyright $\odot 2021$ Rony Kumer Saha. This is an open access article distributed under the Creative Commons Attribution License, which permits unrestricted use, distribution, and reproduction in any medium, provided the original work is properly cited.

\begin{abstract}
In this paper, by exploiting the frequency-domain, we propose a countrywide millimeter-wave (mmWave) spectrum allocation and reuse technique to allocate and reuse spatially the countrywide $28 \mathrm{GHz}$ licensed spectrum and $60 \mathrm{GHz}$ unlicensed spectrum to small cells (SCs) on each floor of a building of each Fifth-Generation (5G) New Radio (NR) Mobile Network Operator (MNO) of an arbitrary country. We develop an interference management scheme, model user statistics per SC, and interferer statistics per apartment and formulate the amount of the $28 \mathrm{GHz}$ and $60 \mathrm{GHz}$ spectra per MNO. We derive average capacity, spectral efficiency (SE), energy efficiency (EE), and cost efficiency (CE) when employing the proposed technique, as well as the traditional static licensed spectrum allocation technique. We discuss the implementation of the proposed technique and evaluate the performance under two scenarios, namely, SCs operate only in the $28 \mathrm{GHz}$ in scenario 1, and both $28 \mathrm{GHz}$ and $60 \mathrm{GHz}$ in scenario 2 . Extensive results and analyses are carried out for four MNOs, i.e., MNOs 1, 2, 3, and 4, in scenario 1. However, in scenario 2, in addition to MNOs 1, 2, 3, and 4, an incumbent Wireless Gigabit (WiGig) operator is considered. It is shown that the proposed technique with no co-channel interference can improve average capacity, SE, EE, and CE of MNO 1 by 3 times, 1.65 times, $75 \%$, and $60 \%$, respectively, in scenario 1, whereas 6.12 times, 5.104 times, $85.8 \%$, and $83.15 \%$, respectively, in scenario 2 . Moreover, with an increase in reuse factors, SE increases linearly and EE increases negative exponentially. Further, we show that the proposed technique can satisfy SE and EE requirements for sixth-generation $(6 \mathrm{G})$ mobile systems. Finally, we discuss offered benefits and point out key issues of the proposed technique for further studies.
\end{abstract}

\section{Introduction}

1.1. Background. The continuing demand for high capacity and data rate in cellular networks due to the growth of mobile devices to serve rich and diverse multimedia contends enforces mobile network operators (MNOs) to redesign the existing networks. The cost and scarcity of the available licensed spectrum are the major concerns toward addressing the high capacity and data rate demand at a low cost per bit transmission in cellular networks. In this regard, due to the license-free access to unlicensed bands and wide spectrum availability in both unlicensed and licensed bands, the operation in millimeter-wave (mmWave) bands is considered as a potential solution to minimize spectrum scarcity and licensing cost for fifth-generation (5G) new radio (NR) and beyond mobile networks. However, to operate in the unlicensed bands, certain regulatory requirements must meet that vary among regions and bands, including, in general, using Listen-Before-Talk (LBT) as a spectrum sharing mechanism, a maximum channel occupancy time, a minimum occupied channel bandwidth requirement, and power limits [1]. Hence, due to regulatory restrictions on the transmission power in the unlicensed bands, like Long Term Evolution-Unlicensed (LTE-U) [2], $5 \mathrm{G}$ NR Unlicensed (5G NR-U) is expected to be deployed in the small cells in the indoor coverage. Accordingly, the $3^{\text {rd }}$ Generation Partnership Project (3GPP) has recently started expanding the operation of cellular networks to the unlicensed bands in 2015 with the Long-Term Evolution (LTE) in 3GPP Release-13 [3]. In this regard, due to wider contiguous bandwidth availability, the $60 \mathrm{GHz}$ unlicensed mmWave band is considered an attractive candidate to operate 5G NR-U. However, the IEEE 802.11-based Wireless Gigabit (WiGig) is in operation in the $60 \mathrm{GHz}$ band. Hence, 
to operate in these unlicensed bands without interfering with each other, an appropriate mechanism is necessary for the cellular networks to coexist with the incumbent WiGig networks.

However, the impact of the operation of mmWave depends on its proper allocation and exploitation that can play a vital role in addressing the spectrum scarcity for an MNO in a country. Spectrum allocation techniques describe how the spectrum specified for a country is allocated to its MNOs. The usefulness of a spectrum allocation technique is affected by factors, such as the amount and duration of the allocated spectrum to an MNO, as well as the user traffic demand of an MNO [4]. By carefully allocating the spectrum specified for a country among its MNOs, the available amount of spectrum for an MNO can be extended considerably. Furthermore, by exploiting the available spectrum for an MNO in space, for example, the utilization of the spectrum can be increased. Accordingly, the spatial reuse of the spectrum to small cells, particularly in a 3-Dimensional (3D) space, e.g., a multistory building, is considered an effective approach to increase the utilization of the available spectrum.

1.2. Related Work. Numerous research studies addressed the coexistence issue of WiFi with cellular networks such as LTE and $5 \mathrm{G}$ NR. Notably, with regard to the LTE and WiFi (LTE/ $\mathrm{WiFi}$ ) coexistence, the authors in [3] elaborated LTE/WiFi coexistence mechanism in time, frequency, and power aspects. Further, the authors in [5] carried out a performance analysis of 3GPP LTE and IEEE 802.11 Wireless Local Area Networks (WLAN) using a fractional bandwidth sharing mechanism. Furthermore, the authors in [2] reviewed the state-of-the-art LTE/WiFi coexistence mechanisms and showed their incorporation into the industry standards. Besides, in [6], the authors studied an effective coexistence mechanism between LTE-U and WiFi systems in the same unlicensed spectrum to enable the cellular network to use LTE- $\mathrm{U}$ while protecting $\mathrm{WiFi}$ access points. In [7], the authors proposed a novel method to allow the coexistence of LTE-U with WiFi networks for $5 \mathrm{G}$ by formulating the problem as maximizing the Quality-of-Experience (QoE) of the LTE-U system and solving it using a game-theoretic approach. Further, the authors in [8] described LTE-U WiFi heterogeneous (HetNet) architecture and outlined the technical challenges for the effective utilization of unlicensed bands in LTE-U WiFi HetNets.

Likewise, regarding the $5 \mathrm{G}$ New Radio on Unlicensed band (NR-U) and WiFi (5G NR-U/WiFi) coexistence, the authors in [9] addressed the coexistence of $5 \mathrm{G}$ NR-U/WiFi in the $6 \mathrm{GHz}$ band and in [10] addressed the coexistence of WiFi with beam-based $5 \mathrm{G}$ NR-U in the mmWave bands. Moreover, in [11], the authors investigated the WiFi and 5G NR coexisted network by implementing a mode selection procedure in the NR base stations to use either the licensed spectrum band or the unlicensed spectrum band. Additionally, in [12], the authors studied the coexistence of WiGig and 5G in Unlicensed spectrum (5G-U) systems in terms of downlink data rate by comparing three different scenarios, including WiGig only, the coexistence of WiGig and $5 \mathrm{G}-\mathrm{U}$ and $5 \mathrm{G}-\mathrm{U}$ only.

Besides, several research studies proposed to use the Almost Blank Subframe- (ABS-) based Enhanced Intercell Interference Coordination (eICIC) technique in LTE to address the coexistence issue between $\mathrm{WiFi}$ and cellular systems in the unlicensed band in the time-domain. For example, by reusing the concept of ABS, the authors in [13] proposed a simple scheme to exist the LTE system with the WiFi system in an unlicensed band and showed an improved throughput per WiFi user performance. Further, the authors in [5] proposed the LTE muting mechanism, where the LTE is kept muting transmission in $n$ of every 5 subframes to allow access to the channel to WiFi users.

Further, numerous research works have already addressed the issues of spectrum allocation as well as spectrum exploitation. For example, the authors in [14] proposed methods for the dynamic spectrum allocation in cognitive radio systems, and the authors in [15] presented a system-level dynamic frequency spectrum allocation scheme based on central heterogeneous network architecture. Using the carrier aggregation technique, the authors in [16] considered the optimization of resource allocation, and the authors in [17] introduced and formulated the problem of the optimum spectrum allocation in cognitive radios. Furthermore, in [18], a new dynamic spectrum allocation algorithm has been proposed to resolve channel conflict problems in channel switching. Regarding the spectrum exploitation by means of reusing the available spectrum, the authors proposed an analytical model to reuse the microwave spectrum in [19], and the $28 \mathrm{GHz}$ mmWave spectrum in [20] in a 3D building of small cells. Likewise, the authors in [21] investigated a number of Fractional Frequency Reuse (FFR) schemes, and the authors proposed the Dynamic Fractional Frequency Reuse (DFFR) method in [22] to reduce the intercell interference.

1.3. Problem Statement and Contribution. Hence, to address the scarcity of available radio spectrum to serve growing demands of high capacity and data rate, as an extension of the LTE Unlicensed (LTE-U) or Licensed Assisted Acces$\mathrm{s}(\mathrm{LAA})$, the $5 \mathrm{G} \mathrm{NR}-\mathrm{U}$ needs to aggregate the $28 \mathrm{GHz}$ or $38 \mathrm{GHz}$ licensed mmWave spectrum and the $60 \mathrm{GHz}$ unlicensed mmWave spectrum [12]. Moreover, to facilitate an improved mmWave spectrum allocation and exploitation, unlike the traditional static licensed spectrum allocation that considers allocating a certain portion of the countrywide spectrum to an MNO, the whole countrywide mmWave spectrum can be allocated to small cells of each MNO to increase its spectrum. Besides, due to the high floor penetration loss, the same countrywide mmWave spectrum for each MNO can be exploited spatially in the interfloor level to reuse the whole spectrum to small cells more than once within a building. Hence, a technique that can aggregate the countrywide full $28 \mathrm{GHz}$ licensed spectrum and $60 \mathrm{GHz}$ unlicensed spectrum and allocate and reuse the aggregated spectrum to small cells of each MNO located on each floor of a building to increase the available spectrum bandwidth to serve on-demand high capacity and data rate is yet to be 
addressed, which we aim to contribute in this paper. In line with so, we contribute the following in this paper:

(i) We propose a countrywide mmWave spectrum allocation and reuse technique to allocate and reuse spatially the countrywide $28 \mathrm{GHz}$ licensed spectrum and $60 \mathrm{GHz}$ unlicensed spectrum to small cells located on each floor of a building of each $5 \mathrm{G}$ NR MNO of an arbitrary country.

(ii) We then develop an interference management scheme, model user statistics per small cell and interferer statistics per apartment, and formulate the amount of the $28 \mathrm{GHz}$ and $60 \mathrm{GHz}$ spectra per MNO.

(iii) We derive average capacity, spectral efficiency (SE), energy efficiency (EE), and cost efficiency (CE) when employing the proposed technique as well as the traditional Static Licensed Spectrum Allocation (SLSA) technique.

(iv) Further, we discuss the implementation of the proposed technique and evaluate the performance under two scenarios, namely, small cells operate only in the $28 \mathrm{GHz}$ in scenario 1 and both $28 \mathrm{GHz}$ and $60 \mathrm{GHz}$ in scenario 2 .

(v) Extensive results and analyses are then carried out for four MNOs, i.e., MNOs 1, 2, 3, and 4, in scenario 1. However, in scenario 2, in addition to MNOs 1, 2, 3, and 4 , an incumbent WiGig operator is considered.

(vi) The performance of the proposed technique with no Co-Channel Interference (CCI) in terms of average capacity, SE, EE, and CE of MNO 1 in scenarios 1 and 2 is evaluated, and the impact of reuse factors on $\mathrm{SE}$ and $\mathrm{EE}$ is shown. Moreover, we show that the proposed technique can satisfy SE and EE requirements for sixth-generation (6G) mobile systems

(vii) Finally, we discuss offered benefits as well as point out key issues of the proposed technique for further studies.

1.4. Organization. In addressing the proposed technique, we first present the system architecture and the proposed technique, as well as develop a frequency-domain CCI management scheme, in Section 2. In Section 3, we model user statistics per small cell and interferer statistics per apartment. We also formulate an expression of the optimal amount of $28 \mathrm{GHz}$ spectrum and $60 \mathrm{GHz}$ spectrum per MNO for an arbitrary number of MNOs in a country. In Section 4, we derive average capacity, SE, EE, and CE when employing the proposed technique, as well as the traditional SLSA technique. In addition, we show mathematically the outperformance of the proposed technique over the SLSA technique and discuss the implementation of the proposed technique. In Section 5, we evaluate the performance under two scenarios, namely, small cells operate only in the $28 \mathrm{GHz}$ in scenario 1 and both $28 \mathrm{GHz}$ and $60 \mathrm{GHz}$ in scenario 2 . Extensive results and analyses are carried out by varying the effect of the spectrum reuse and the co-channel interference of interferer user equipments (UEs) within each apartment for four MNOs, i.e., MNOs 1, 2, 3, and 4, in scenario 1. However, in scenario 2 , in addition to MNOs $1,2,3$, and 4, an incumbent WiGig operator is considered. Moreover, we also show that the proposed technique with two extreme CCI scenarios, including no CCI and the maximum CCI, for an $\mathrm{MNO}$, can achieve the SE and EE requirements for $6 \mathrm{G}$ mobile systems in both scenarios. Section 6 covers the discussion on the offered benefits of the proposed technique, as well as its further research directions. We conclude the paper in Section 7. In Appendix A, a list of acronyms/abbreviations is shown in Table 1, and a list of selected notations is given in Table 2.

1.5. Declaration. This paper was presented partly as a conference article [23] that received the "Best Paper Award" at the Fifteenth International Conference on Systems and Networks Communications (ICSNC), Porto, Portugal, 2020. Unlike the conference version [23] that addresses the allocation and reuse of the $28 \mathrm{GHz}$ licensed mmWave spectrum only, the scope of this journal version is extended to the allocation and reuse of both the $28 \mathrm{GHz}$ licensed spectrum and $60 \mathrm{GHz}$ unlicensed spectrum. Further, as compared to [23], we model user statistics per small cell and interferer statistics per apartment in a building and formulate the amount of the $28 \mathrm{GHz}$ and $60 \mathrm{GHz}$ spectra per MNO. We show mathematically the outperformance of the proposed technique over the traditional SLSA technique and discuss the implementation of the proposed technique. We evaluate the performance of the proposed technique under two scenarios, including small cells operate only in the $28 \mathrm{GHz}$ as in the case of [23], and small cells operate in both $28 \mathrm{GHz}$ and $60 \mathrm{GHz}$. A more detailed performance evaluation and analysis than that in [23] are carried out by varying the effect of the spectrum reuse (both vertically within a building and horizontally inbetween buildings) and the co-channel interference (by considering all possible numbers of interferer UEs within each apartment). Finally, in addition to the offered benefits discussed in [23], the future research directions of the proposed technique are also discussed here. Because the paper was presented partly in [23], some materials in this paper, in terms of, e.g., text, equations, figures, tables, notations, and abbreviations, may be found merged with that in [23] with relevant citations. However, this paper is rewritten by defining a set of new problems and presenting their solutions along with additional results and findings such that the readers will find it self-contained, detailed, and insightful with a minimum level of commonality with its conference version [23].

\section{System Architecture, Proposed Technique, and Interference Management}

2.1. System Architecture. We consider a system architecture that consists of an arbitrary number of MNOs and WiGig operators countrywide. Each MNO has three types of base stations (BSs), namely, macrocell BSs (MBSs), Picocell BSs (PBSs), and Small-Cell BSs (SBSs). Hence, we show only one $\mathrm{MNO}$, i.e. MNO 1, in Figure 1(a) of a total of four MNOs countrywide, for example. MBSs and PBSs are located outdoors and serve only macrocell UEs (MUs). Small cell 
TABLE 1: List of acronyms/abbreviations.

\begin{tabular}{lc}
\hline Acronym/abbreviation & Definition \\
\hline 3D & 3 -Dimensional \\
6 G & Sixth-generation \\
BS & Base station \\
CCI & Co-channel interference \\
CE & Cost efficiency \\
EE & Energy efficiency \\
FFR & Fractional frequency reuse \\
ISD & Intersite distance \\
LOS & Line-of-sight \\
mmWave & Millimeter-wave \\
MNO & Mobile network operator \\
Non-LOS & NLOS \\
NRA & National regulatory agency \\
PBS & Picocell base station \\
RB & Resource block \\
SBS & Small cell base station \\
SE & Spectral efficiency \\
SLSA & Static licensed spectrum allocation \\
TTI & Transmission time interval \\
UE & User equipment \\
\hline
\end{tabular}

TABLE 2: List of selected notations.

\begin{tabular}{|c|c|}
\hline Notation & Description \\
\hline$t$ & Index of a TTI \\
\hline$T$ & Simulation run time with the maximum tin \\
\hline$O$ & Number of MNOs of a country \\
\hline$o$ & Index of an MNO \\
\hline$M$ & Amount of mmWave spectrum per MNO in SLSA \\
\hline$l$ & Index of a building \\
\hline$L$ & Number of buildings per macrocell \\
\hline$i$ & Index of an $\mathrm{RB}$ \\
\hline$P_{\mathrm{MC}}, P_{\mathrm{PC}}$, and $P_{\mathrm{SC}}$ & The transmission power of a macrocell, a picocell, and a small cell, respectively, of an MNO $o$ \\
\hline$\omega_{\mathrm{FL}}$ & Number of floors in a building \\
\hline$\omega_{\mathrm{fl}}$ & Index of a floor in a building \\
\hline$M_{\mathrm{C}}$ & Countrywide mmWave spectrum in RBs \\
\hline$t_{\text {rnw }}$ & Licensed renew term \\
\hline$S_{F, o}$ & Number of SBSs in any building $l$ for an MNO $o$ \\
\hline$\varepsilon_{C}$ & Cost of the countrywide $28 \mathrm{GHz}$ mmWave spectrum $M_{C}$ \\
\hline$\varepsilon_{o}$ & Spectrum licensing fee paid by an MNO $o$ \\
\hline$N_{o}$ & Number of subscribers of an MNO $o$ at term $t_{\text {rnw }}$ \\
\hline$N_{C}$ & Number of subscribers of a country at term $t_{\mathrm{rnw}}$ \\
\hline$M_{o, t}^{l, \omega_{\mathrm{fl}}}$ & $\begin{array}{l}\text { The optimal amount of licensed spectrum in RBs for an MNO } o \text { on any floor } \omega_{\mathrm{fl}} \text { in a building } l \text { in TTI } \\
\qquad t \text { at term } t_{\mathrm{rnw}}\end{array}$ \\
\hline$\sigma_{t, i, o}(\cdot)$ & A link throughput at $\mathrm{RB}=i$ in $\mathrm{TTI}=t$ for an $\mathrm{MNO} o$ at $t_{\mathrm{rnw}}$ in bps per $\mathrm{Hz}$ \\
\hline$\rho_{t, i, o}^{l, o}(\cdot)$ & A link SINR at $\mathrm{RB}=i$ in TTI $=t$ for an MNO $o$ at $t_{\text {rnw }}$ in $\mathrm{dB}$ \\
\hline$M_{\mathrm{MBS}, o}$ & Spectrum in RBs of a macrocell for an $\mathrm{MNO} o$ \\
\hline $\begin{array}{l}\sigma_{28, O}^{\mathrm{CP}}(\cdot), \sigma_{28, O}^{\mathrm{SE}}(\cdot), \sigma_{28, O}^{\mathrm{EE}}(\cdot), \text { and } \\
\sigma_{28, O}^{\mathrm{CE}}(\cdot)\end{array}$ & $\begin{array}{l}\text { System-level average capacity, SE, EE, and CE, respectively, for all MNOs } O \text { countrywide at } t_{\mathrm{rnw}} \text { for } \\
\qquad l=L \text { when small cells of all MNOs operating in the } 28 \mathrm{GHz}\end{array}$ \\
\hline $\begin{array}{l}\sigma_{60, O}^{\mathrm{CP}}(\cdot), \sigma_{60, O}^{\mathrm{SE}}(\cdot), \sigma_{60, O}^{\mathrm{EE}}(\cdot), \text { and } \\
\sigma_{60, O}^{\mathrm{CE}}(\cdot)\end{array}$ & $\begin{array}{l}\text { System-level average capacity, SE, EE, and CE, respectively, for all MNOs } O \text { countrywide at } t_{\mathrm{rnw}} \text { for } \\
\qquad l=L \text { when small cells of all MNOs operating in the } 60 \mathrm{GHz}\end{array}$ \\
\hline $\begin{array}{l}\sigma_{\mathrm{CA}, O}^{\mathrm{CP}}(\cdot), \sigma_{\mathrm{CA}, O}^{\mathrm{SE}}(\cdot), \sigma_{\mathrm{CA}, O}^{\mathrm{EE}}(\cdot), \text { and } \\
\sigma_{\mathrm{CA}, O}^{\mathrm{CE}}(\cdot)\end{array}$ & $\begin{array}{l}\text { System-level average capacity, } \mathrm{SE}, \mathrm{EE} \text {, and CE, respectively, for all MNOs } O \text { countrywide at } t_{\mathrm{rnw}} \text { for } \\
\qquad l=L \text { when small cells of all MNOs operating in the } 28 \mathrm{GHz} \text { and } 60 \mathrm{GHz}\end{array}$ \\
\hline $\begin{array}{l}\sigma_{\mathrm{SLS}, O}^{\mathrm{CP}}(\cdot), \sigma_{\mathrm{SLSA}, O}^{\mathrm{SE}}(\cdot), \sigma_{\mathrm{SLSA}, O}^{\mathrm{EE}}(\cdot), \text { and } \\
\sigma_{\mathrm{SLSA}, O}^{\mathrm{CE}}(\cdot)\end{array}$ & $\begin{array}{l}\text { System-level average capacity, SE, EE, and CE, respectively, for all MNOs } O \text { countrywide at } t_{\mathrm{rnw}} \text { for } \\
\qquad l=L \text { when small cells of all MNOs operating in SLSA }\end{array}$ \\
\hline$\varsigma_{28, O, I F}^{\mathrm{CP}}, \varsigma_{28, \mathrm{O}, \mathrm{IF}}^{\mathrm{SE}}, \varsigma_{28, \mathrm{O}, \mathrm{IF}}^{\mathrm{EE}}$, and $\varsigma_{28, \mathrm{O}, \mathrm{IF}}^{\mathrm{CE}}$ & Average capacity, SE, EE, and CE improvement factors, respectively, due to operating in the $28 \mathrm{GHz}$ \\
\hline
\end{tabular}




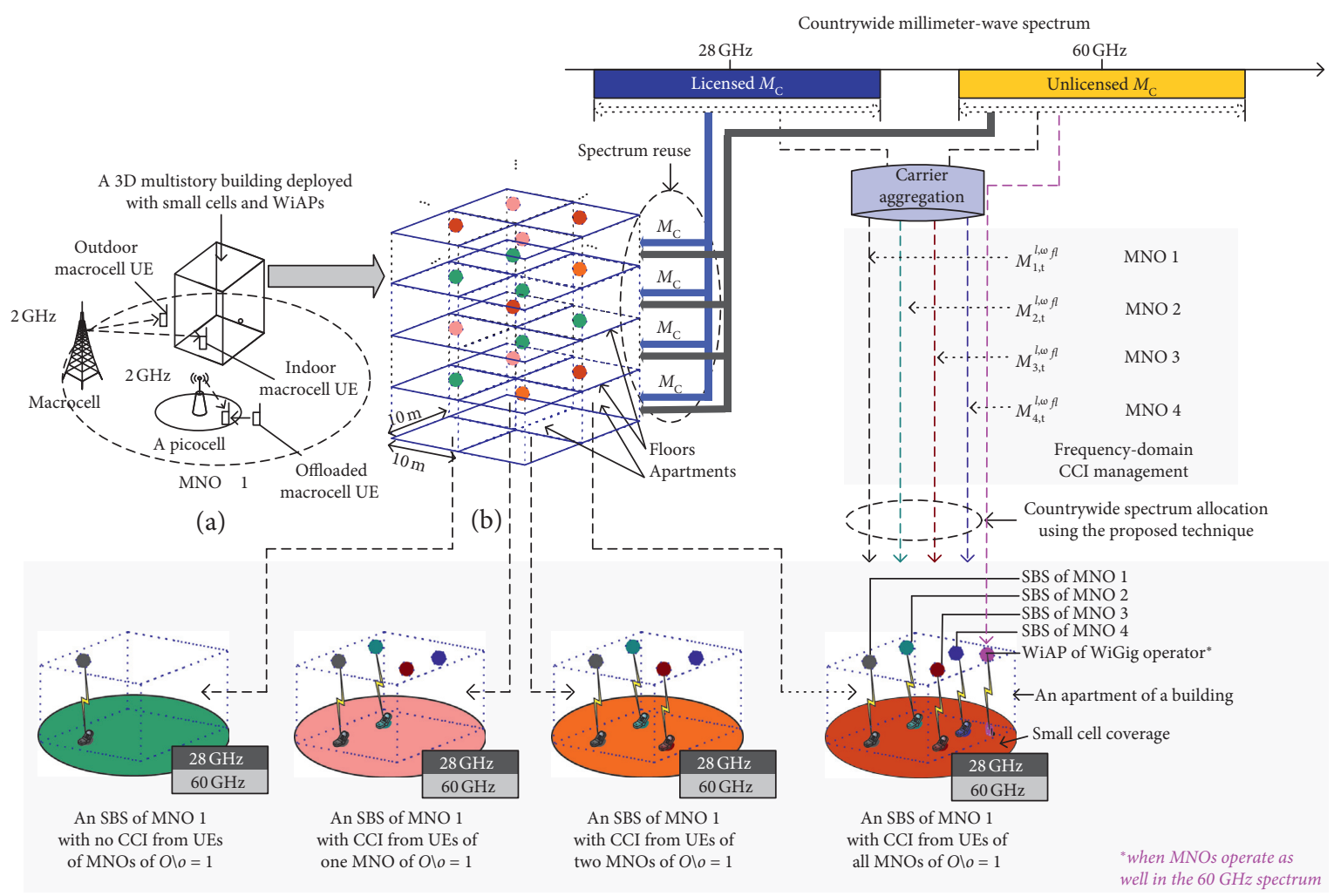

(c)

FIGURE 1: A system architecture consisting of four MNOs and a single WiGig operator countrywide.

BSs (SBSs) of each MNO are dual-band enabled and deployed only within multistory buildings in an urban environment that serve only their UEs within buildings. Because of favorable characteristics, MBSs and PBSs operate in the $2 \mathrm{GHz}$ spectrum band to cover a large area to reduce handoff occurrences, whereas SBSs operate only in the $28 \mathrm{GHz}$ and/or $60 \mathrm{GHz}$ mmWave bands to cover a small area at high data rates.

Each MNO is allowed to get access to the countrywide full $28 \mathrm{GHz}$ spectrum subject to avoiding co-channel interference (CCI). Figure 1(c) shows all possible combinations of co-channel interferers $(0,1,2$, and 3 for four MNOs) for a small cell of MNO 1. However, when small cells operate in the $60 \mathrm{GHz}$ unlicensed spectrum, additional CCI may occur due to the WiGig Access Points (WiAPs) of any incumbent WiGig operator. In such a case, each small cell, by employing carrier aggregation technology, can operate in both the $28 \mathrm{GHz}$ licensed and $60 \mathrm{GHz}$ unlicensed bands to extend its available spectrum bandwidth opportunistically. Moreover, due to a high floor penetration loss, both mmWave signals get attenuated significantly such that in practice CCI effect from one floor to its adjacent ones can be considered negligible. Due to this reason, the same countrywide spectrum can be reused to small cells of each MNO on each floor of a multistory building as shown in Figure 1(b), resulting in improving the system performances further. Hence, by allowing to get access to the full countrywide $28 \mathrm{GHz}$ and $60 \mathrm{GHz}$ mmWave spectrum bands and reusing the same spectrum to small cells on each floor, both spectrum allocation and spectrum exploitation of mmWave can be obtained, a technique of which we propose in the following section.

2.2. Proposed Technique. Unlike the traditional licensed spectrum allocation techniques that mostly allocate statically a portion of the total spectrum assigned to a country to each MNO, we propose to allocate the full $28 \mathrm{GHz}$ licensed mmWave spectrum specified for a country to each of its MNOs to operate its small cells deployed on each floor in a building subject to avoiding CCI from one MNO to another. In addition, by exploiting the high floor penetration loss of the $28 \mathrm{GHz}$ mmWave signal, the allocated spectrum to each MNO can further be reused to its small cells on each floor of a multistory building. The licensing fee for the full countrywide spectrum can be distributed among all MNOs by charging each MNO fairly such that the spectrum licensing fee for an MNO is updated in accordance with the change in its number of subscribers at each license renewal term $t_{\text {rnw }}$.

The above technique can also be applied if small cells of an MNO operate in an unlicensed mmWave band except that the additional CCI from the incumbent WiFi technologies needs to be taken into account. In this regard, assume that small cells of MNOs operate in the $60 \mathrm{GHz}$ unlicensed band, in addition to MNOs, CCI from the incumbent WiGig operators must be considered. We discuss the CCI avoidance mechanism and the corresponding impact on the mmWave spectrum allocated to 
each MNO in both the $28 \mathrm{GHz}$ licensed and $60 \mathrm{GHz}$ unlicensed bands in the following sections.

\subsection{Co-Channel Interference Management}

(1) $28 \mathrm{GHz}$ licensed mmWave spectrum: small cells of all MNOs located on each floor within a building operate in the same countrywide $28 \mathrm{GHz}$ licensed mmWave spectrum. Hence, due to the coexistence of UEs of all MNOs on the same floor, CCI occurs if small-cell UEs of more than one MNO are scheduled to the licensed spectrum simultaneously. CCI can be avoided by allocating UEs of small cells on a floor orthogonally in the frequency-domain such that UEs of MNOs located on the same floor are allocated to different parts of the $28 \mathrm{GHz}$ licensed spectrum as shown in Figure 2 [24]. In other words, small-cell UEs of not more than one MNO can be allocated to the same frequency in any transmission time interval (TTI). The existence of an interfering UE can be detected by the small cell itself using any conventional spectrum sensing techniques to avoid simultaneous access to the same spectrum.

(2) $60 \mathrm{GHz}$ unlicensed mmWave spectrum: unlike the $28 \mathrm{GHz}$ licensed mmWave spectrum, since IEEE 802.11 technologies operate in the unlicensed bands, e.g., $2.4 \mathrm{GHz}, 5 \mathrm{GHz}$, and $60 \mathrm{GHz}$ globally, CCI can be originated from the incumbent IEEE 802.11ad/ay, also termed as WiGig, operating in the $60 \mathrm{GHz}$ unlicensed mmWave spectrum. Hence, in addition to CCI originated from the peer MNOs, CCI from the WiGig operators needs to be taken into account to operate small cells of any $\mathrm{MNO}$ in the $60 \mathrm{GHz}$ unlicensed mmWave band. In this regard, like the $28 \mathrm{GHz}$ licensed mmWave band, following the above procedure as shown in Figure 2, CCI due to operating small cells in the $60 \mathrm{GHz}$ spectrum can also be avoided in the frequency-domain by considering all WiGig operators, as well as all MNOs, operating in each apartment on any floor of a building.

\section{Mathematical Analysis}

3.1. Modeling User Statistics per Small Cell. In this section, we are interested in finding the number of users per small cell of an $\mathrm{MNO} o$ in an apartment for an observation time Q. Let $U_{s} \in \mathbf{U}_{s}=\left\{0,1,2, \ldots, U_{s, \max }\right\}$ denote the number of UEs served by an SBS $s$ of an MNO $o$ at any time $t$. According to $[25,26]$, sessions or call arrivals can be modeled as a Poisson process. Hence, the traffic activity of a small cell UE served by an in-building SBS can be modeled as an exponentially distributed continuous-time Poisson process. Since given the present state, the future state is independent of the past state, the traffic activity of a UE can be modeled as a twostate Markov chain where the off-state traffic activity to onstate traffic activity transition rate of a UE is denoted by $\lambda$ and the on-state traffic activity to off-state traffic activity transition rate is denoted by $\mu$.
Let $p(0), p(1), p(2), \ldots, p\left(U_{s, \max }\right)$ denote on-state probabilities of an SBS $s$ (corresponding to the number of active UEs $U_{s}$ ) in an apartment. The values of these probabilities can be found following the Birth-Death process [25] as shown in Figure 3 . Let $\lambda_{U_{s}}$ and $\mu_{U_{s}}$ denote, respectively, the birth rate and the death rate. Then, according to $[27,28]$, the following holds:

$$
\begin{aligned}
& \lambda_{U_{s}}= \begin{cases}\left(U_{s, \max }-U_{s}\right) \lambda, & 0 \leq U_{s} \leq U_{s, \max }, \\
0, & \text { otherwise, }\end{cases} \\
& \mu_{U_{s}}=U_{s} \times \mu .
\end{aligned}
$$

Hence, the probability of any $U_{s}$ can be given by

$$
p\left(U_{s}\right)=p(0)\left(\left(\frac{\lambda}{\mu}\right)^{U_{s}} \times\left(\begin{array}{c}
U_{s, \max } \\
U_{s}
\end{array}\right)\right) .
$$

But,

$$
\sum_{U_{s}=0}^{U_{s, \max }} p\left(U_{s}\right)=1
$$

Then, using (2) and (3), the following can be obtained:

$$
p(0)=\frac{1}{(1+(\lambda / \mu))^{U_{s, \max }}} .
$$

Now from (2), we can find the following. In other words, the probability that $U_{s}$ number of UEs are served by an SBS $s$ is given by

$$
p\left(U_{s}\right)=\frac{U_{s, \max } !}{U_{s} !\left(U_{s, \max }-U_{s}\right) !} \times\left(\frac{\lambda}{\mu}\right)^{U_{s}} \times \frac{1}{(1+(\lambda / \mu))^{U_{s, \max }}} .
$$

Hence, the expected value of the number of UEs served simultaneously at any time $t$ is then given by

$$
E\left[U_{s}\right]=\sum_{U_{s}=0}^{U_{s}=U_{s, \max }}\left(U_{s} \times p\left(U_{s}\right)\right)
$$

Note that, since the rate of arrival of UEs to any inbuilding SBS $s$ is relatively low due to the small coverage, the value of $U_{s}$ is small in the Poisson distribution of small cell UEs. In other words, smaller values of $U_{s}$ are more probable than the larger ones such that the distribution of small cell UEs of an SBS lies mostly toward the left of the curve.

3.2. Optimal Amount of Spectrum per MNO. Let $O$ denote the maximum number of MNOs of a country such that $o \in \mathbf{O}=\{1,2, \ldots, O\}$. Let $S_{F, o}$ denote the total number of small cells of an MNO $o$ in any building $l \in \mathbf{L}=\{1,2,3$, $\ldots, L\}$ such that $s_{x, o} \in \mathbf{S}_{\mathbf{x}, \mathbf{o}}=\left\{0,1,2, \ldots, S_{F, o}\right\}$. Denote $M_{\mathrm{C}}$ as the countrywide total amount of mmWave spectrum in the $28 \mathrm{GHz}$ licensed band, as well as $60 \mathrm{GHz}$ unlicensed band, defined in terms of the number of Resource Blocks (RBs) where an $\mathrm{RB}$ is equal to $180 \mathrm{kHz}$. Assume that $E\left[U_{s, o}\right]=1$, i.e., each small cell $s_{x, o}$ of an MNO $o$, can serve the maximum of one UE at a time. 


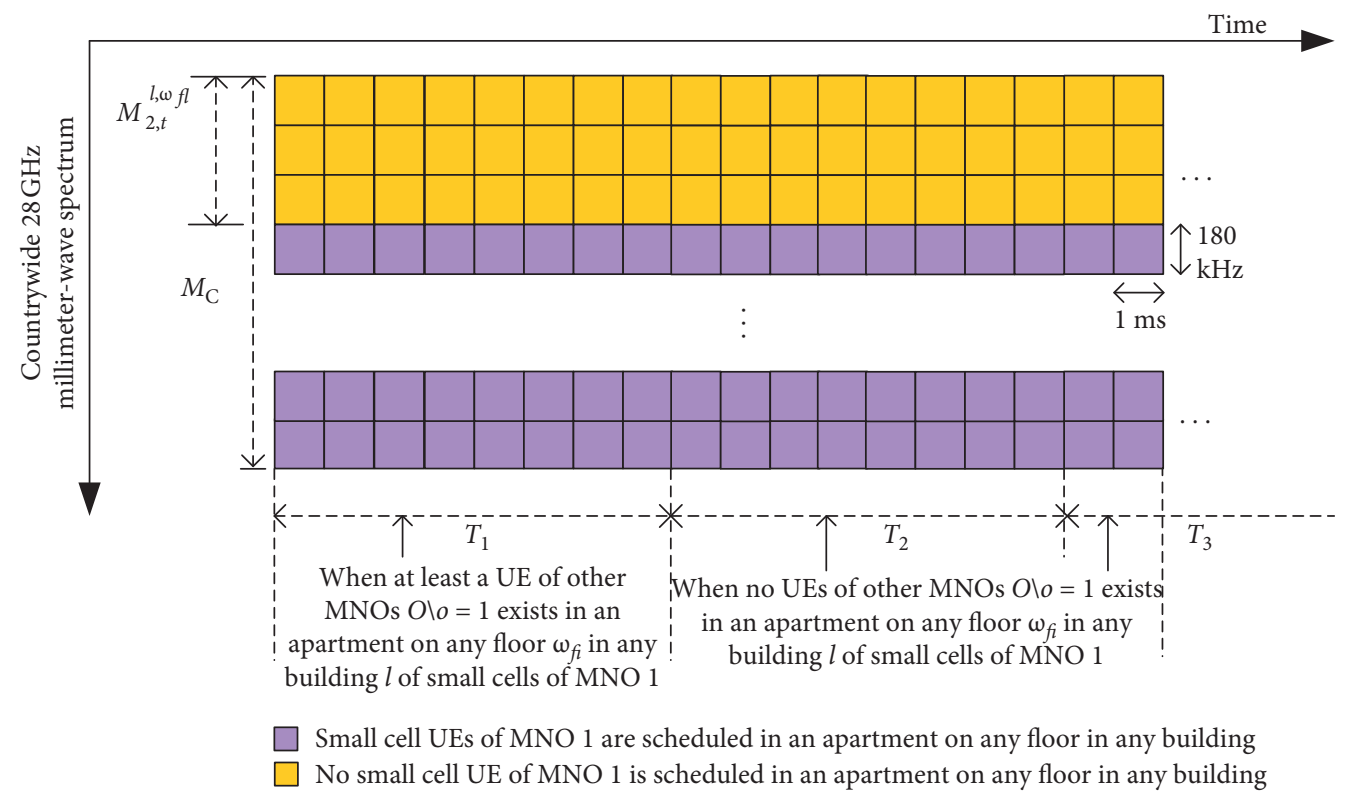

FIgURE 2: Frequency-domain CCI avoidance technique for UEs of MNO 1 in an apartment on any floor $\omega_{f l}$ in a building l. $T_{1}, T_{2}$, and $T_{3}$ define arbitrary and equal observation time intervals within $|\mathbf{T}|=Q[23]$.

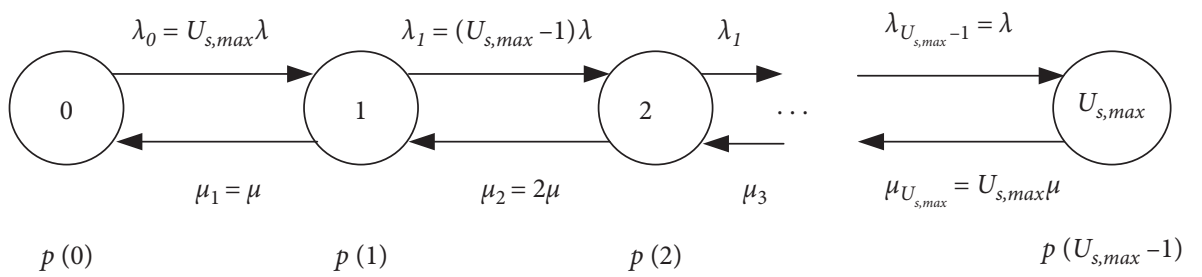

FIGURE 3: Occupancy or traffic in-progress state diagram for UEs per small cell in an apartment.

Let $N_{o}$ denote the total number of subscribers of an MNO $o$ such that $\sum_{o}^{O} N_{o} \leq N_{C}$ where $N_{C}$ denotes the maximum number of subscribers of a country at any term $t_{\text {rnw }}$. Also, UEs of not more than one MNO $o$ on the same floor $\omega_{f l}$ can be served by the same RB in any TTI $t$ of a building $l$. The amount of spectrum allocated to UEs of an MNO $o$ on a floor $\omega_{\mathrm{fl}}$ in a building $l$ at term $t_{\mathrm{rnw}}$ in TTI $t$ is defined as follows.

"The amount of $28 \mathrm{GHz}$ licensed spectrum or $60 \mathrm{GHz}$ unlicensed spectrum allocated to UEs of an MNO $o$ on any floor $\omega_{\mathrm{fl}}$ of a building $l$ at term $t_{\text {rnw }}$ is defined in accordance with the ratio of the number of subscribers $N_{o}$ of MNO $o$ to the sum of the total number of subscribers $N_{t}^{l, \omega_{f l}}$ of all MNOs $O$ corresponding to the same floor $\omega_{\mathrm{fl}}$ of a building $l$ in any TTI $t$ at term $t_{\text {rnw. }}$.

Note that the radio spectrum is not free of cost. Hence, licensing more spectra causes an increase in the cost of an MNO. Moreover, as the total amount of spectrum specified for a country is fixed, licensing more spectrum by one MNO causes scarcity of the required spectrum by another MNO in a country, resulting in degrading the quality-of-service (QoS). This problem can be addressed if each MNO takes license of the amount of the spectrum as low as possible corresponding to its actual number of subscribers so that the issue of the underutilized or unused spectrum by one MNO, as well as the lack of a sufficient amount of spectrum for another MNO, to serve its necessary user demand can be addressed.

Since each MNO favors minimizing the cost of licensing spectrum while ensuring to serve its user demands adequately to retain QoS, we consider a minimization problem for allocating the countrywide mmWave spectrum to each MNO to increase the overall countrywide mmWave spectrum utilization. Hence, the optimal amount of either $28 \mathrm{GHz}$ or $60 \mathrm{GHz}$ mmWave spectra $M_{o, t}^{l, \omega_{\mathrm{fl}}}$ in RBs for an MNO $o \in \mathbf{O}$ on any floor $\omega_{f l}$ of a building $l$ in TTI $t$ at a renewal term $t_{\text {rnw }}$ can be found by solving the following problem:

$$
\begin{aligned}
& \min _{o \in \mathbf{O}} M_{o, t}^{l, \omega_{f l}} \\
& \text { (a) } \frac{N_{o}}{N_{t}^{l, \omega_{\mathrm{fl}}}}=\frac{M_{o, t}^{l, \omega_{\mathrm{fl}}}}{M_{\mathrm{C}}}
\end{aligned}
$$

subject to

$$
\text { (b) } \forall o \forall t_{\text {rnw }} \sum_{o}^{O} N_{o} \leq N_{\mathrm{C}} \text {. }
$$

The solution to the above optimization problem can be expressed as follows and is given in proof: 


$$
M_{o, t}^{l, \omega_{\mathrm{f}^{*}}}=\left[\left(\left(\frac{N_{o}}{\sum_{o=1}^{O}\left(1_{v_{o}}\left(N_{o}\right) \times N_{o}\right)}\right) \times M_{C}\right)\right] .
$$

Proof. The solution to the optimization problem in (7) can be found as follows. In general, the number of subscribers of all MNOs is not the same at any $t_{\text {rnw }}$. Hence, assume that $N_{1}>N_{2}>\cdots>N_{O}$ at $t_{\text {rnw }}$ such that the constraint $7(\mathrm{~b})$ is satisfied. Since a UE of any MNO O $\backslash$ o in any TTI may not exist on any floor $\omega_{\mathrm{fl}}$ of a building $l$ of small cells of an MNO $o, N_{t}^{l, \omega_{\mathrm{fl}}}$ can be expressed for an arbitrary value of $O=4$ as

$$
N_{t}^{l, \omega_{\mathrm{fl}}}=\sum_{o=1}^{O}\left(1_{v_{o}}\left(N_{o}\right) \times N_{o}\right)
$$

where $v_{o} \in\left\{N_{1}, N_{2}, N_{3}, N_{4}\right\} .1(\cdot)$ defines that $1(\cdot)=1$ if $N_{o}$ exists in the set $\nu_{o}$; otherwise, $1(\cdot)=0$.

Since the number of RBs is strictly an integer, using (9), and the constraint 7(a), the optimal value of $M_{o, t}^{l, \omega_{\mathrm{fl}}}$ is given by

$$
\begin{aligned}
& M_{o, t}^{l, \omega_{\mathrm{f}}{ }^{*}}=\left(\frac{N_{o}}{N_{t}^{l, \omega_{\mathrm{fl}}}}\right) \times M_{C}, \\
& M_{o, t}^{l, \omega_{\mathrm{f}} *}=\left[\left(\left(\frac{N_{o}}{\sum_{o=1}^{O}\left(1_{v_{o}}\left(N_{o}\right) \times N_{o}\right)}\right) \times M_{C}\right)\right] .
\end{aligned}
$$

Note that if a UE of any MNO $\mathbf{O} \backslash o$ in any TTI $t$ on any floor $\omega_{\mathrm{fl}}$ of a building $l$ does not exist, then $N_{t}^{l, \omega_{\mathrm{fl}}}=N_{o}$ in (9), which results in $M_{o, t}^{l, \omega_{\mathrm{f}}^{*}}=M_{C}$. This implies that the whole countrywide $28 \mathrm{GHz}$ or $60 \mathrm{GHz}$ mmWave spectrum can be allocated in all TTIs $t$ to UEs of small cells of an MNO $o$ on any floor $\omega_{\mathrm{fl}}$ in a building $l$. The same process described above is applicable for all MNOs $o \in \mathbf{O}$ at each renewal term $t_{\text {rnw }}$ to update $M_{o, t}^{l, \omega_{\mathrm{fl}}}$ in any TTI $t$ to avoid CCI. Hence, using (8), the countrywide $28 \mathrm{GHz}$ or $60 \mathrm{GHz}$ spectra can be reused to small cells of each $\mathrm{MNO} o$ on any floor $\omega_{\mathrm{fl}}$ in a building $l$ to improve countrywide $28 \mathrm{GHz}$ and $60 \mathrm{GHz}$ mmWave spectrum utilization. Further, the higher the number of subscribers $N_{o}$ of an MNO $o$ at term $t_{\text {rnw }}$, the greater the amount of mmWave spectrum $M_{o, t}^{l, \omega_{\mathrm{fl}}}$ allocated to MNO $o$ on any floor $\omega_{\mathrm{fl}}$ in a building $l$ in any TTI $t$ at term $t_{\text {rnw }}$.

3.3. Modeling Interferer Statistics per Apartment. Recall that we assume $E\left[U_{s, o}\right]=1$ for each SBS of each MNO $o$ within each apartment of any building. Since the arrival of a UE to any SBS in an apartment can be modeled as a Poisson process, under the above assumption, the presence of the number of interferer UEs within each apartment can be expressed following the same procedure presented for modeling the user statistics per small cell in Section 3.1. In doing so, let $U_{k} \in U_{\mathbf{k}}=\left\{0,1,2, \ldots, U_{k \text {, max }}\right\}$ denote the number of interferer UEs served by each SBS of MNOs $\mathbf{O} \backslash$ o in any TTI $t$ for an SBS $s$ of MNO $o$, where $U_{k, \max }=O-1$.
Then, the probability of $U_{k}$ number of interferer UEs for an SBS $s$ of MNO $o$ in an apartment is given by

$$
p\left(U_{k}\right)=\frac{U_{k, \max } !}{U_{k} !\left(U_{k, \max }-U_{k}\right) !} \times\left(\frac{\lambda}{\mu}\right)^{U_{k}} \times \frac{1}{(1+(\lambda / \mu))^{U_{k, \max }}} .
$$

Hence, the expected value of the number of interferer UEs served simultaneously in any time $t$ in an apartment for an SBS $s$ of MNO $o$ is then given by

$$
E\left[U_{k}\right]=\sum_{U_{k}=0}^{U_{k}=U_{k, \max }}\left(U_{k} \times p\left(U_{k}\right)\right)
$$

From (12), it can be found that the maximum amount of spectrum is allocated to an MNO $o$ when no interferer UEs of MNOs $\mathbf{O} \backslash o$ exist within an apartment on any floor (i.e., $\left.E\left[U_{k}\right]=0\right)$. Likewise, the minimum amount of spectrum is allocated to an MNO $o$ when each interferer UE of MNOs $\mathbf{O}$ lo exist within each apartment on any floor, i.e., $E\left[U_{k}\right]=\left(U_{k, \max }-1\right)$.

\section{Performance Metrics Estimation and Analysis}

\subsection{Performance Metrics}

4.1.1. $28 \mathrm{GHz}$ Licensed Spectrum. Let $S_{\mathrm{M}, \mathrm{o}}$ denote the number of macrocells and $S_{\mathrm{P}, \mathrm{o}}$ denote the number of picocells per macrocell of an MNO $o$. Also, let $\mathbf{T}$ denote the simulation run time with the maximum time of $Q$ (in time step each lasting $1 \mathrm{~ms}$ ) such that $\mathbf{T}=\{1,2,3, \ldots, \mathrm{Q}\}$. Let $P_{\mathrm{MC}}$, $P_{\mathrm{PC}}$, and $P_{\mathrm{SC}}$ denote, respectively, the transmission power of a macrocell, a picocell, and a small cell of an MNO $o$. Using Shannon's capacity formula, a link throughput at $\mathrm{RB}=i$ in $\mathrm{TTI}=t$ for an MNO $o$ at $t_{\text {rnw }}$ in bps per Hz is given by [29]:

$$
\sigma_{t, i, o}\left(\rho_{t, i, o}\right)=\left\{\begin{array}{cl}
0 & \rho_{t, i, o}<-10 \mathrm{~dB} \\
\beta \log _{2}\left(1+10^{\left(\rho_{t, i, o}(\mathrm{~dB}) / 10\right)}\right) & -10 \mathrm{~dB} \leq \rho_{t, i, o} \leq 22 \mathrm{~dB} \\
4.4 & \rho_{t, i, o}>22 \mathrm{~dB}
\end{array}\right\},
$$

where $\beta$ denotes the implementation loss factor.

Let $M_{\mathrm{MBS}, o}$ denote the spectrum in RBs of a macrocell for an MNO $o$. Then, the total capacity of all macrocell UEs for an MNO $o$ at $t_{\text {rnw }}$ can be expressed as

$$
\sigma_{\mathrm{MBS}, o}=\sum_{t=1}^{Q} \sum_{i=1}^{M_{\mathrm{MBS}, o}} \sigma_{t, i, o}\left(\rho_{t, i, o}\right)
$$

where $\sigma$ and $\rho$ are responses over $M_{\mathrm{MBS}, o}$ RBs of all macroUEs in $t \in \mathbf{T}$ for an MNO $o$ at $t_{\text {rnw }}$. If all SBSs $s_{\omega_{\mathrm{f}}, o}$ on any floor $\omega_{\mathrm{fl}}$ in a building $l$ of an MNO $o$ serves simultaneously in all TTI $t \in \mathbf{T}$ in the $28 \mathrm{GHz}$ licensed spectrum, then the aggregate capacity served by an SBS, all SBSs per floor $\omega_{\mathrm{fl}}$, as well as all SBSs on all floors $\omega_{\mathrm{FL}}$ in a building $l$, of an MNO $o$ at a renewal term $t_{\text {rnw }}$ are given, respectively, by 


$$
\begin{aligned}
\sigma_{28, o, s_{x, o}}^{l, \omega_{\mathrm{fl}}} & =\sum_{t \in T} \sum_{i=1}^{M_{o, t}^{l, \omega_{\mathrm{f}}}} \sigma_{t, i, o}\left(\rho_{t, i, o}\right), \\
\sigma_{28, o, s_{\omega_{\mathrm{fl}}, o}}^{l, \omega_{\mathrm{fl}}} & =\sum_{s_{x, o}=1}^{S_{\omega_{\mathrm{f}}, o}} \sigma_{28, o, s_{x, o}}^{l, \omega_{\mathrm{f}}}, \\
\sigma_{28, o}^{l, \omega_{\mathrm{FL}}} & =\sum_{\omega_{\mathrm{fl}}=1}^{\omega_{\mathrm{FL}}} \sigma_{28, o, s_{\omega_{\mathrm{fl}}, o}}^{l, \omega_{\mathrm{fl}}} .
\end{aligned}
$$

Due to a short distance between a small cell UE and its SBS and a low transmission power of an SBS, we assume similar indoor signal propagation characteristics for all $L$ buildings per macrocell for an MNO $o$ at $t_{\text {rnw }}$. Then, by linear approximation, the system-level average aggregate capacity, $\mathrm{SE}$, and $\mathrm{EE}$ for all MNOs $\mathrm{O}$ operating in the $28 \mathrm{GHz}$ countrywide at $t_{\text {rnw }}$ for $l=L$ can be given by

$$
\sigma_{28, O}^{\mathrm{CP}}(L)=\sum_{o=1}^{O}\left(\sigma_{\mathrm{MBS}, o}+\left(L \times \sigma_{28, o}^{l, \omega_{\mathrm{FL}}}\right)\right) .
$$

Since $\left(L \times \sigma_{28, o}^{l, \omega_{\mathrm{FL}}}\right) \gg \sigma_{\mathrm{MBS}, o}$, roughly, (18) can be given by

$$
\begin{aligned}
\sigma_{28, O}^{\mathrm{CP}}(L) & \cong \sum_{o=1}^{O}\left(L \times \sigma_{28, o}^{l, \omega_{\mathrm{FL}}}\right), \\
\sigma_{28, O}^{\mathrm{SE}}(L) & =\frac{\sigma_{28, O}^{\mathrm{CP}}(L)}{\left(\left(M_{\mathrm{C}}+\sum_{o=1}^{O} M_{\mathrm{MBS}, o}\right) \times Q\right)}, \\
\sigma_{28, O}^{\mathrm{EE}}(L) & =\frac{\left(\sum_{o=1}^{O}\left(\begin{array}{c}
\left(L \times S_{F, o} \times P_{\mathrm{SC}}\right) \\
+\left(S_{P, o} \times P_{\mathrm{PC}}\right)+\left(S_{M, o} \times P_{\mathrm{MC}}\right)
\end{array}\right)\right)}{\left(\sigma_{28, O}^{\mathrm{CP}}(L) / Q\right)},
\end{aligned}
$$

where $S_{F, o}=\sum_{\omega_{\mathrm{fl}}=1}^{\omega_{\mathrm{FL}}} s_{\omega_{\mathrm{f}}, o}$ denotes the total number of SBSs in any building $l$ for an $\mathrm{MNO} o$.
4.1.2. $60 \mathrm{GHz}$ Unlicensed Spectrum. When small cells of all MNOs operate only in the $60 \mathrm{GHz}$ unlicensed mmWave spectrum, the maximum number of operators in an apartment is equal to the sum of all MNOs $\mathrm{O}$ (when operating in the $28 \mathrm{GHz}$ licensed band) and WiGig operators. Since the value of $\mathrm{O}$ does not change, the above equations from (7) to (21) for the $28 \mathrm{GHz}$ licensed spectrum are equally applicable for the $60 \mathrm{GHz}$ unlicensed spectrum to find the average capacity $\sigma_{60, O}^{\mathrm{CP}}(L), \operatorname{SE} \sigma_{60, O}^{\mathrm{SE}}(L)$, and $\mathrm{EE} \sigma_{60, O}^{\mathrm{EE}}(L)$ of small cells of all MNOs when operating in the $60 \mathrm{GHz}$ spectrum using (18), (20), and (21), respectively.

Note that even though $\mathrm{O}$ does not change when small cells of MNOs operate in the $60 \mathrm{GHz}$, if any WiAP coexists with small cells of MNOs in an apartment, the amount of unlicensed spectrum allocated to WiAPs is given by (8), i.e., in accordance with the percentage of the number of subscribers of WiGig operators with respect to that of all MNOs O. In other words, unlike in case of operating in the $28 \mathrm{GHz}$ licensed spectrum, the existence of any WiAP causes not to allocate the full countrywide unlicensed spectrum $M_{\mathrm{C}}$ to MNOs $\mathrm{O}$ since some percentage of the unlicensed spectrum $M_{\mathrm{C}}$ is allocated to WiAPs. Note that even if the number of UEs of small cells is too high or too low, each operator (either a WiGig or an MNO) is allocated to a fair amount of unlicensed spectrum defined by (8) such that UEs of neither an MNO nor a WiGig operator get deprived of being allocated to the $60 \mathrm{GHz}$ unlicensed spectrum. Moreover, like LTE muting, the proposed technique mutes the transmission of small cells of MNOs on the unlicensed spectra defined by (8) to allow the transmission of only WiAPs of WiGig operators to avoid their unexpected blockage and provide fair access to the unlicensed spectrum.

4.1.3. $28 \mathrm{GHz}$ Licensed and $60 \mathrm{GHz}$ Unlicensed Spectra. If small cells are enabled with both the $28 \mathrm{GHz}$ and $60 \mathrm{GHz}$ mmWave bands using the Carrier Aggregation (CA) technology such that each small cell of an MNO can operate in both the $28 \mathrm{GHz}$ and $60 \mathrm{GHz}$ spectrum bands, the average capacity of small cells in (18) is given by the sum of the capacities achieved by both the $28 \mathrm{GHz}$ and $60 \mathrm{GHz}$ mmWave spectra as given below:

$$
\begin{aligned}
& \sigma_{\mathrm{CA}, O}^{\mathrm{CP}}(L)=\left(\sigma_{28, O}^{\mathrm{CP}}(L)+\sigma_{60, O}^{\mathrm{CP}}(L)\right), \\
& \sigma_{\mathrm{CA}, O}^{\mathrm{SE}}(L)=\frac{\sigma_{\mathrm{CA}, O}^{\mathrm{CP}}(L)}{\left(\left(M_{C}+\sum_{o=1}^{O} M_{\mathrm{MBS}, o}\right) \times Q\right)}, \\
& \sigma_{\mathrm{CA}, O}^{\mathrm{EE}}(L)=\frac{\left(\sum_{o=1}^{O}\left(\left(L \times S_{F, o} \times P_{\mathrm{SC}}\right)+\left(S_{P, o} \times P_{\mathrm{PC}}\right)+\left(S_{M, o} \times P_{\mathrm{MC}}\right)\right)\right)}{\left(\sigma_{\mathrm{CA}, O}^{\mathrm{CP}}(L) / Q\right)} .
\end{aligned}
$$


Note that due to paying a spectrum license fee for the $28 \mathrm{GHz}$ spectrum and no license fee for the $60 \mathrm{GHz}$ spectrum, in defining SE above when small cells operate in the $60 \mathrm{GHz}$ spectrum as well, we consider the $28 \mathrm{GHz}$ licensed spectrum as the effective spectrum.

4.1.4. Traditional SLSA Technique. In the traditional SLSA technique, a fair allocation of the licensed mmWave spectrum to each MNO in a country is assumed, i.e., each MNO is given a license exclusively for an equal amount of the mmWave spectrum of $M$ RBs such that for $O=4$, $M_{C}=4 M$. Now, using (17)-(21), the system-level average capacity, SE, and EE of small cells of all MNOs $O$ countrywide when operating in the $28 \mathrm{GHz}$ spectrum at $t_{\mathrm{rnw}}$ for $l=L$ can be given by

$$
\begin{gathered}
\sigma_{\mathrm{SLSA}, O}^{\mathrm{CP}}(L)=\sum_{o=1}^{O}\left(\sigma_{\mathrm{MBS}, o}+\left(L \times \sum_{\omega_{\mathrm{fl}}=1}^{\omega_{\mathrm{FL}}} \sum_{s_{x, o}=1}^{S_{\omega_{\mathrm{f}}, o}} \sum_{t \in \mathbf{T}} \sum_{i=1}^{M} \sigma_{s_{x, o}, t, i, o}^{\omega_{\mathrm{fl}}}\left(\rho_{s_{x, o}, t, i, o}^{\omega_{\mathrm{fl}}}\right)\right),\right. \\
\sigma_{\mathrm{SLSA}, O}^{\mathrm{CP}}(L) \cong \sum_{o=1}^{O}\left(L \times \sum_{\omega_{\mathrm{fl}}=1}^{\omega_{\mathrm{FL}}} \sum_{s_{x, o}=1}^{S_{\omega_{\mathrm{fl}}, o}} \sum_{t \in \mathbf{T}} \sum_{i=1}^{M} \sigma_{s_{x, o}, t, i, o}^{\omega_{\mathrm{fl}}}\left(\rho_{s_{x, o}, t, i, o}^{\omega_{\mathrm{fl}}}\right)\right), \\
\sigma_{\mathrm{SLSA}, O}^{\mathrm{SE}}(L)=\frac{\sigma_{\mathrm{SLSA}, O}^{\mathrm{CP}}(L)}{\left(\left(M_{C}+\sum_{o=1}^{O} M_{\mathrm{MBS}, o}\right) \times Q\right)}, \\
\sigma_{\mathrm{SLSA}, O}^{\mathrm{EE}}(L)=\frac{\left(\sum_{o=1}^{O}\left(\left(L \times S_{F, o} \times P_{\mathrm{SC}}\right)+\left(S_{P, o} \times P_{\mathrm{PC}}\right)+\left(S_{M, o} \times P_{\mathrm{MC}}\right)\right)\right)}{\left(\sigma_{\mathrm{SLSA}, O}^{\mathrm{CP}}(L) / Q\right)} .
\end{gathered}
$$

4.1.5. Cost Efficiency. Let $\varepsilon_{C}$ denote the cost of the countrywide $28 \mathrm{GHz}$ licensed mmWave spectrum $M_{C}$. Recall that an $\mathrm{MNO} o$ pays the spectrum licensing fee based on its number of subscribers $N_{o}$ at $t_{\text {rnw }}$ with respect to that of all MNOs $N_{C}$. Assume that an MNO $o$ pays the spectrum licensing fee of $\varepsilon_{o}$ corresponding to $N_{o}$ at $t_{\text {rnw }}$ such that $\varepsilon_{o}$ can be given by

$$
\varepsilon_{o}=\left(\frac{N_{o}}{N_{C}}\right) \times \varepsilon_{C} .
$$

Now, define Cost Efficiency (CE) as the cost required per unit achievable average capacity (i.e., per bps) such that the $\mathrm{CE}$ at term $t_{\text {rnw }}$ can be expressed as follows when small cells operate in the $28 \mathrm{GHz}$ licensed spectrum.

$$
\varsigma_{28, O}^{\mathrm{CE}}=\frac{\varepsilon_{C}}{\sigma_{28, O}^{\mathrm{CP}}(L)} .
$$

Likewise, for SLSA, CE can be expressed as follows:

$$
\varsigma_{\mathrm{SLSA}, O}^{\mathrm{CE}}=\frac{\varepsilon_{C}}{\sigma_{\mathrm{SLSA}, O}^{\mathrm{CP}}(L)} \text {. }
$$

Note that, the $60 \mathrm{GHz}$ unlicensed mmWave spectrum is exempted from paying licensing fees such that when small cells operate only in the $60 \mathrm{GHz}$ unlicensed spectrum, CE is theoretically zero, representing an ideal state. However, if small cells operate in both the $28 \mathrm{GHz}$ licensed spectrum and $60 \mathrm{GHz}$ unlicensed spectrum, CE can be expressed as follows:

$$
\varsigma_{\mathrm{CA}, O}^{\mathrm{CE}}=\frac{\varepsilon_{C}}{\sigma_{\mathrm{CA}, O}^{\mathrm{CP}}(L)} .
$$

\subsection{Performance Improvement}

4.2.1. Performance Improvement Factors. The factors representing an improvement in average capacity, SE, EE, and $\mathrm{CE}$ due to the $28 \mathrm{GHz}$ licensed mmWave spectrum can be expressed, respectively, as follows:

$$
\begin{gathered}
\varsigma_{28, \mathrm{O}, \mathrm{IF}}^{\mathrm{CP}}=\frac{\sigma_{28, O}^{\mathrm{CP}}(L)}{\sigma_{\mathrm{SLSA}, O}^{\mathrm{CP}}(L)}, \\
\varsigma_{28, \mathrm{O}, \mathrm{IF}}^{\mathrm{SE}}=\frac{\sigma_{28, O}^{\mathrm{SE}}(L)}{\sigma_{\mathrm{SLSA}, O}^{\mathrm{SE}}(L)}, \\
\varsigma_{28, \mathrm{O}, \mathrm{IF}}^{\mathrm{EE}}=\frac{\sigma_{28, O}^{\mathrm{EE}}(L)}{\sigma_{\mathrm{SLSA}, O}^{\mathrm{EE}}(L)}, \\
\varsigma_{28, \mathrm{O}, \mathrm{IF}}^{\mathrm{CE}}=\frac{\varsigma_{28, O}^{\mathrm{CE}}}{\varsigma_{\mathrm{SLSA}, O}^{\mathrm{CE}}} .
\end{gathered}
$$

Now, following (31)-(33), we can express average capacity, SE, and EE improvement factors when small cells operate either in only the $60 \mathrm{GHz}$ spectrum or in both the $28 \mathrm{GHz}$ and $60 \mathrm{GHz}$ spectra. However, because $\mathrm{CE}$ is zero when small cells operate in only the $60 \mathrm{GHz}$ unlicensed spectrum, the CE improvement factor is also zero. This implies that no further improvement in CE can be possible due to reaching an ideal state. However, if small cells operate in both the $28 \mathrm{GHz}$ and $60 \mathrm{GHz}$ bands, $\mathrm{CE}$ improvement factor can be given by 


$$
\varsigma_{\mathrm{CA}, \mathrm{O}, \mathrm{IF}}^{\mathrm{CE}}=\frac{\varsigma_{\mathrm{CA}, \mathrm{O}}^{\mathrm{CE}}}{\sigma_{\mathrm{SLSA}, O}^{\mathrm{CE}}(L)} .
$$

4.2.2. Performance Improvement Analysis. In the following, we analyze the outperformance of the proposed technique over the traditional SLSA technique in terms of average capacity, SE, EE, and CE. In doing so, we consider the countrywide subscriber statistics of all MNOs on any floor $\omega_{\mathrm{fl}}$ in a building $l$ at a renewal term $t_{\text {rnw }}$ in time $t$. Assume that the number of countrywide subscribers of MNO 1, MNO 2, MNO 3, and MNO 4 is, respectively, $10 \%, 20 \%$, $30 \%$, and $40 \%$ of the total number of subscribers in a country. Since the spectrum is allocated to any MNO $o$ in proportionate to its number of subscribers, using (8), the allocated spectra to MNO 1, MNO 2, MNO 3, and MNO 4, are given, respectively, by $M_{1, t}^{l, \omega_{\mathrm{fl}}}=\left(0.1 \times M_{\mathrm{C}}\right), M_{2, t}^{l, \omega_{\mathrm{fl}}}=(0.2$ $\left.\times M_{\mathrm{C}}\right), M_{3, t}^{l, \omega_{\mathrm{fl}}}=\left(0.3 \times M_{\mathrm{C}}\right)$, and $M_{4, t}^{l, \omega_{\mathrm{fl}}}=\left(0.4 \times M_{\mathrm{C}}\right)$.

Hence, the total amount of the spectrum used to serve user demands of all MNOs when employing the proposed technique is given by

$$
\begin{aligned}
& M_{O, t}^{l, \omega_{\mathrm{f}}, \text { Prop }}=\left(0.1 \times M_{C}\right)+\left(0.2 \times M_{C}\right)+\left(0.3 \times M_{C x}\right)+\left(0.4 \times M_{C}\right), \\
& M_{O, t}^{l, \omega_{\mathrm{f}} \text {,Prop }}=M_{C} .
\end{aligned}
$$

On the other hand, when employing the traditional SLSA technique, each MNO $o$ is allocated to an equal amount of spectrum of $\left(0.25 \times M_{C}\right)$. However, the number of subscribers of MNO 1, MNO 2, MNO 3, and MNO 4 is not the same such that each MNO does not need the same amount of spectrum. More specifically, since the amount of spectrum required to serve the user demand of any $\mathrm{MNO}$ varies in accordance with its number of subscribers, MNO 1, MNO 2, MNO 3, and MNO 4 can use, respectively, 10\%, 20\%, 25\%, and $25 \%$ of $M_{C}$. Hence, the total amount of the spectrum used to serve user demands of all MNOs when employing the SLSA technique is given by

$$
\begin{aligned}
& M_{O, t}^{l, \omega_{\mathrm{f}}, \mathrm{SL}}=\left(0.1 \times M_{C}\right)+\left(0.2 \times M_{C}\right)+\left(0.25 \times M_{C}\right)+\left(0.25 \times M_{C}\right), \\
& M_{O, t}^{l, \omega_{\mathrm{f}}, \mathrm{SL}}=0.8 \times M_{C} .
\end{aligned}
$$

From (36) and (37), we can write the following:

$$
M_{O, t}^{l, \omega_{\mathrm{fl}}, \operatorname{Prop}}>M_{O, t}^{l, \omega_{\mathrm{fl}}, \mathrm{SL}} \text {. }
$$

Since the achievable capacity is directly proportional to the spectrum bandwidth, the following relation holds:

$$
\sigma_{O, t}^{l, \omega_{\mathrm{fl}}, \operatorname{Prop}}>\sigma_{\mathrm{O}, t}^{l, \omega_{\mathrm{fl}}, \mathrm{SL}},
$$

where $\sigma_{O, t}^{l, \omega_{\mathrm{fl}}, \text { Prop }} \propto M_{O, t}^{l, \omega_{\mathrm{f}}, \text { Prop }}$ and $\sigma_{O, t}^{l, \omega_{\mathrm{f}}, \mathrm{SL}} \propto M_{O, t}^{l, \omega_{\mathrm{f}}, \mathrm{SL}}$ denote, respectively, the achievable capacities corresponding to the spectra in (36) and (37) due to employing the proposed and SLSA techniques.

Since SE is directly proportional, whereas EE and CE are inversely proportional, to the achievable capacity, using (20) and (25) for the SE, (21) and (26) for the EE, and (28) and (29) for the CE, it can be shown that the proposed technique provides better $\mathrm{SE}, \mathrm{EE}$, and $\mathrm{CE}$ performances than the traditional SLSA technique.

4.3. Implementation Perspectives. The implementation of the proposed technique warrants the following issues, including updating the dynamic usage of the countrywide spectrum in the $28 \mathrm{GHz}$ and $60 \mathrm{GHz}$ on each floor by UEs of different MNOs and the WiGig operator and enforcing CCI management. In this regard, SBSs of each MNO per floor can keep sensing to detect the status of the shared full countrywide spectrum usage and coordinate with SBSs of other MNOs on the same floor to update the CCI status and amount of the shared spectrum usage for each MNO. However, such coordination among SBSs of different MNOs generates control signaling overheads depending on the size of the group of the coordinated SBSs. The larger the size of the group of the coordinated SBSs, the greater the amount of generated control signaling overheads, as well as delay in updating the CCI status.

In general, depending on the architectural characteristics, coordination can be performed in two ways, namely, centralized and distributed manners [30]. In centralized coordination (CEC) of multiple MNOs sharing the same spectrum, instead of coordinating one MNO to another directly, MNOs coordinate with each other via a central entity, e.g., spectrum controller, which can be the National Regulatory Agency (NRA), that controls sharing of the common spectrum among MNOs. Typically, CEC is preferable for static spectrum sharing schemes such that updating spectrum usage status on a real-time basis is not necessary [30], i.e., the spectrum sharing among MNOs is performed on a long-term basis. Though CEC is easy to control and can provide fair and reliable spectrum allocation, it suffers from the necessity of additional network infrastructure, the long delay from real-time traffic transmissions, as well as large control signaling overheads due to dynamic spectrum usage.

Unlike CEC, in distributed coordination (DIC), MNOs coordinate with each other directly in a distributed manner [30]. The presence of interferers can be detected on-demand using spectrum sensing techniques to improve system performance locally by avoiding CCI. Though it suffers from the reliable detection of idle bands and the reduction in overall data transmission time due to the flexible qualityof-service (QoS) requirements, DIC is suitable for MNOs to coexist with WiFi systems. Hence, based on the pros and cons of centralized and distributed coordination approaches, we present a hybrid centralized-distributed coordination approach to implement the proposed spectrum sharing technique using both coordination approaches as given below.

The implementation is divided into two parts, interMNO countrywide that does not require frequent spectrum usage updates and intersmall cell of MNOs that requires frequent spectrum usage updates. In inter-MNO countrywide, since the allocated spectrum $M_{o, t}^{l, \omega_{\mathrm{fl}}}$ to each MNO $o$ is 


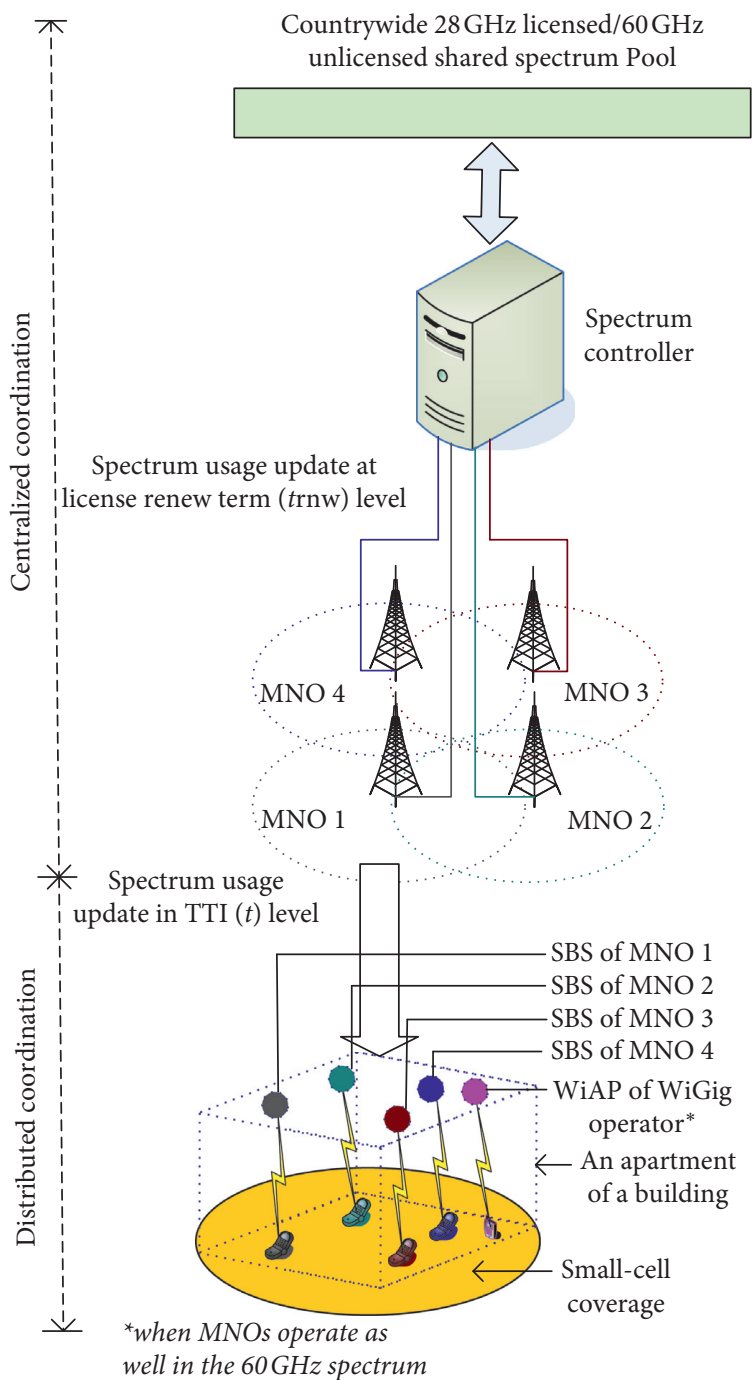

FIGURE 4: Illustration of implementation of the proposed technique using a hybrid centralized-distributed coordination approach.

TABLE 3: Default parameters and assumptions.

\begin{tabular}{|c|c|}
\hline Parameters and assumptions & Value \\
\hline $28 \mathrm{GHz}$ and $60 \mathrm{GHz}$ spectra cou & $200 \mathrm{MHz}$ (each) \\
\hline $\begin{array}{l}\text { Number of MNOs, WiGig operators, and } \\
\text { subscribers }\end{array}$ & 4,1 , and $N_{C}$ \\
\hline $\begin{array}{l}\text { Number of subscribers for MNOs 1, 2, 3, and } 4 \text {, } \\
\text { respectively } \\
\text { For each MNO }\end{array}$ & $\begin{array}{c}40 \%, 30 \%, 20 \% \text {, and } 10 \% \text { of } N_{C}\left(28 \mathrm{GHz} \text { only) } 35 \%, 25 \%, 15 \%, 10 \% \text { and } 20 \% \text { of } N_{C} \text { (both }\right. \\
28 \mathrm{GHz} \text { and } 60 \mathrm{GHz})\end{array}$ \\
\hline E-UTRA simulation case ${ }^{1}$ & $3 \mathrm{GP}$ \\
\hline $\begin{array}{l}\text { Cellular layout }{ }^{2} \text {, intersite distance }(\mathrm{ISD})^{1,2}, \\
\text { transmit direction }\end{array}$ & Hexagonal grid, dense urban, 3 secto \\
\hline Carrier freq & $\begin{array}{r}2 \mathrm{GHz} \text { non-line-of-sight (NLOS) for macro } \\
\text { for all sr }\end{array}$ \\
\hline Number of cells & 1 macrocell, 2 picocells, $280 \mathrm{~s}$ \\
\hline Total BS transmit power ${ }^{1}(\mathrm{dBm})$ & $\begin{array}{l}46 \text { for macrocell }{ }^{1}, 37 \text { for picocells }{ }^{1}, 19 \text { for small cells operating in } 28 \mathrm{GHz}^{1,3}, 17.3 \text { for small } \\
\text { cells operating in } 60 \mathrm{GHz}^{5}\end{array}$ \\
\hline Co-channel small-scale fading model ${ }^{1,3,4,5}$ & Frequency selective Rayleigh for $2 \mathrm{GHz}$, none for $28 \mathrm{GHz}$ and $60 \mathrm{GHz}$ \\
\hline
\end{tabular}


TABLE 3: Continued.

\begin{tabular}{|c|c|c|c|}
\hline \multicolumn{3}{|c|}{ Parameters and assumptions } & Value \\
\hline \multirow{5}{*}{$\begin{array}{l}\text { Path } \\
\text { loss }\end{array}$} & \multirow{2}{*}{ MBS and a $\mathrm{UE}^{1}$} & Outdoor macrocell & \multirow{2}{*}{$P L(\mathrm{~dB})=15.3+37.6 \log _{10} R, R$ is in $\mathrm{m}$} \\
\hline & & UE & \\
\hline & MBS and a UE & $\begin{array}{l}\text { Indoor macrocell } \\
\mathrm{UE}\end{array}$ & $P L(\mathrm{~dB})=15.3+37.6 \log _{10} R+L_{\text {ow }}, R$ is in $\mathrm{m}$ \\
\hline & \multicolumn{2}{|c|}{ PBS and a UE ${ }^{1}$ UE } & $P L(\mathrm{~dB})=140.7+36.7 \log _{10} R, R$ is in $\mathrm{km}$ \\
\hline & \multicolumn{2}{|l|}{$\mathrm{SBS}_{4,5}$ and $\mathrm{a} \mathrm{UE}^{1,2}$} & 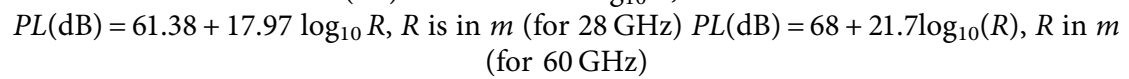 \\
\hline \multicolumn{3}{|c|}{ Lognormal shadowing standard deviation $(\mathrm{dB})$} & 8 for $\mathrm{MBS}^{2}, 10$ for $\mathrm{PBS}^{1}$, and 9.9 for SBS$(\text { for } 28 \mathrm{GHz})^{2,}, 4$, and $0.88(\text { for } 60 \mathrm{GHz})^{5}$ \\
\hline \multicolumn{3}{|c|}{ Antenna configuration } & Single-input single-output for all BSs and UEs \\
\hline \multicolumn{3}{|c|}{ Antenna pattern (horizontal) } & Directional $\left(120^{\circ}\right)$ for $\mathrm{MBS}^{1}$, omnidirectional for $\mathrm{PBS}^{1}$ and $\mathrm{SBS}^{1}$ \\
\hline \multicolumn{3}{|c|}{ Antenna gain plus connector loss $(\mathrm{dBi})$} & 14 for $\mathrm{MBS}^{2}, 5$ for $\mathrm{PBS}^{1}, 5$ for $\mathrm{SBS}^{1,3}$ \\
\hline \multicolumn{3}{|c|}{ UE antenna gain $2,3,5$} & $0 \mathrm{dBi}$ (for $2 \mathrm{GHz}$ ), $5 \mathrm{dBi}$ (for $28 \mathrm{GHz}$ ), $5 \mathrm{dBi}$ (for $60 \mathrm{GHz}$ biconical horn) \\
\hline \multicolumn{3}{|c|}{ UE noise figure $^{2,3,5}$ and UE speed ${ }^{1}$} & $9 \mathrm{~dB}$ (for $2 \mathrm{GHz}$ ) and $10 \mathrm{~dB}$ (for $28 \mathrm{GHz}$ and $60 \mathrm{GHz}$ ), $3 \mathrm{~km} / \mathrm{hr}$ \\
\hline \multicolumn{3}{|c|}{$\begin{array}{l}\text { Picocell coverage, number of macrocell UEs, } \\
\text { and macrocell UEs offloaded to all picocells }{ }^{1}\end{array}$} & $40 \mathrm{~m}$ (radius), $30,2 / 15$ \\
\hline \multicolumn{3}{|c|}{ Indoor macrocell UEs ${ }^{1}$} & $35 \%$ \\
\hline \multicolumn{3}{|c|}{$\begin{array}{l}\text { 3D multistory building and SBS models } \\
\text { (square-grid apartments): number of buildings, } \\
\text { number of floors per building, number of } \\
\text { apartments per floor, number of SBSs per } \\
\text { apartment, number of SBSs per building, area } \\
\text { of an apartment, materials used }\end{array}$} & $L, 35,8,1,280,10 \times 10 \mathrm{~m}^{2}$, reinforced concrete \\
\hline \multicolumn{3}{|c|}{ Scheduler and traffic model ${ }^{2}$} & Proportional fair and full buffer \\
\hline \multicolumn{3}{|c|}{ Type of SBSs } & Closed subscriber group femtocell BSs \\
\hline \multicolumn{3}{|c|}{ Channel state information } & Ideal \\
\hline \multicolumn{3}{|c|}{$\mathrm{TTI}^{1}$ and scheduler time constant $\left(t_{c}\right)$} & $1 \mathrm{~ms}$ and $100 \mathrm{~ms}$ \\
\hline \multicolumn{3}{|c|}{ Total simulation run time } & $8 \mathrm{~ms}$ \\
\hline
\end{tabular}

Taken ${ }^{1}$ from [31], ${ }^{2}$ from [32], ${ }^{3}$ from [33], from ${ }^{4}$ [34], from ${ }^{5}$ [35].

updated at each term, $t_{\mathrm{rnw}}$, which is relatively a long time, CEC is suitable to reallocate $M_{o, t}^{l, \omega_{\mathrm{fl}}}$ to each MNO $o$ at any $t_{\mathrm{rnw}}$ with limiting control signaling overheads and overall low latency as shown in Figure 4. However, in the intersmall cell of different MNOs within an apartment on any floor of a building, spectrum usage status of SBSs of all MNOs along with WiAPs of the WiGig operator is updated real-time basis in every TTI level (which is a very short time of $1 \mathrm{~ms}$ ) such that DIC is suitable to reallocate the spectrum (both licensed and unlicensed) to each SBS of each MNO per apartment (Figure 4).

\section{Performance Evaluation}

5.1. Default Parameter and Assumption. Table 3 shows the default simulation parameters and assumptions used for evaluating the performance of the proposed technique for all MNOs $O$ countrywide. The performance metrics are derived analytically for an arbitrary number of MNOs in a country, and the evaluation is carried out for four MNOs (i.e., $O=4$ ) as an example scenario. The arrival of mobile traffic to a small cell is captured using the Poisson process to model the presence of UEs of MNOs within each apartment. Simulation assumptions and parameters used for the performance evaluation are in line with the recommendations from the standardization bodies such as 3GPP and International Telecommunication UnionRadiocommunication Sector (ITU-R).
For simplicity in analysis and finding a closed-form expression, we assume that each small cell of an MNO $o$ can serve one UE at a time, i.e., $E\left[U_{s, o}\right]=1$. Moreover, because of the favorable signal propagation characteristics in indoor environments and the availability of large spectrum bandwidth, we consider the $28 \mathrm{GHz}$ licensed mmWave spectrum and $60 \mathrm{GHz}$ unlicensed mmWave spectrum bands to serve high data rates within a short distance. However, all the macrocells and picocells are considered operating at the $2 \mathrm{GHz}$ band to provide large coverage and fewer hand-offs. Because high-frequency signals exhibit a low multipath fading effect indoors, the Line-Of-Sight (LOS) large-scale signal propagation model is assumed for the $28 \mathrm{GHz}$ and $60 \mathrm{GHz}$ spectra. Further, due to serving a UE at a short distance over a LOS channel by an SBS, similar signal propagation characteristics are considered within the same building, as well as between adjacent buildings.

Because of a high external wall penetration loss, low transmission power, and the existence of distance-dependent path loss for the distance in-between adjacent buildings for high-frequency signals, we assume an insignificant CCI effect from SBSs of one building to that of adjacent buildings resulting in reusing the same spectrum to SBSs within each building. Furthermore, we adopt the full buffer traffic model to consider serving user traffic at all the time and the proportional fair scheduler to provide a balanced trade-off between the fairness and throughput performances. The performance results are generated by a simulator built using the computational tool MATLAB R2012b taking into 


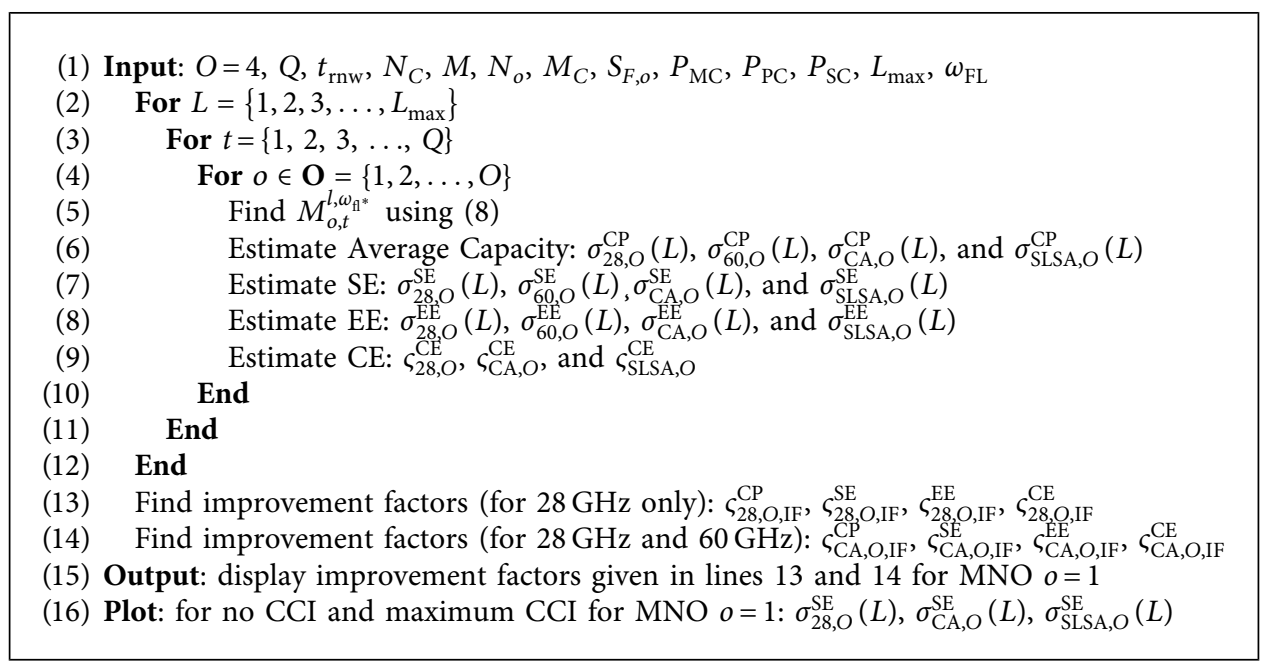

Algorithm 1: Proposed technique.

account all parameters and assumptions stated above and given in Table 3. Finally, the algorithm used to generate the performance results is given in Algorithm 1.

5.2. Performance Result and Analysis. The performance of the proposed technique is evaluated for two spectrum scenarios, including the $28 \mathrm{GHz}$ licensed band, as well as $60 \mathrm{~Hz}$ unlicensed band, with regard to the traditional SLSA technique. When operating only in the $28 \mathrm{GHz}$ licensed band, we assume that MNO 1, MNO 2, MNO 3, and MNO 4 have the number of subscribers of $40 \%, 30 \%, 20 \%$, and $10 \%$, respectively, of the total number of subscribers countrywide $N_{C}$ at any term $t_{\text {rnw }}$ (Table 3 ). However, when small cells of an MNO operate as well in the $60 \mathrm{GHz}$ unlicensed band, due to the presence of the incumbent WiAPs that may cause CCI to small cells, in addition to four MNOs, we assume that a single WiGig operator coexists with MNOs in each apartment. Accordingly, we assume that the WiGig operator has the number of subscribers of $20 \%$ of the total number of subscribers countrywide such that a subscriber base of $30 \%$, $25 \%, 15 \%$, and $10 \%$, respectively, of MNO 1, MNO 2, MNO 3 , and MNO 4, is assumed (as an example scenario) when small cells are operating in the $60 \mathrm{GHz}$ unlicensed band, along with the $28 \mathrm{GHz}$ licensed band.

5.2.1. Impact of the $60 \mathrm{GHz}$ Unlicensed Spectrum and CCI. In both spectrum scenarios, we consider UEs of MNO 1 to evaluate the performance of the proposed technique by varying the number of co-channel interferer UEs of $I_{\mathrm{CCI}}=0$ to $I_{\mathrm{CCI}}=3$ of MNOs $\mathbf{O} \backslash o=1$ per apartment on a single floor of a building for a country with $O=4 \mathrm{MNOs}$. Figure 5 shows the performance improvement of the proposed technique in terms of the average capacity, $\mathrm{SE}, \mathrm{EE}$, and $\mathrm{CE}$ with respect to that of the traditional SLSA technique. Note that in (8), $I_{\mathrm{CCI}}=1$ corresponds to a UE of $\mathrm{MNO}=2$ with $30 \%$ of subscribers, and $I_{\mathrm{CCI}}=2$ corresponds to UEs of $\mathrm{MNO}=2$ plus $\mathrm{MNO}=3$ with $20 \%$ of subscribers. Whereas $I_{\mathrm{CCI}}=0$ and $I_{\mathrm{CCI}}=3$ correspond to two extreme scenarios, defining, respectively, when no CCI occurs due to the absence of UEs of all other MNOs $\mathbf{O} \backslash o=1$, and when the maximum CCI occurs due to the presence of UEs of each MNO of $\mathbf{O} \backslash o=1$ on a floor in a building.

With no co-channel interferer UEs in any apartment (i.e., $I_{\mathrm{CCI}}=0$ ), the maximum amount of full countrywide mmWave spectrum can be allocated to SBSs of MNO 1 on each floor in all TTIs. As $\mathrm{I}_{\mathrm{CCI}}$ increases, the amount of allocated spectrum to MNO 1 decreases, and for the maximum co-channel interferer UEs, $I_{\mathrm{CCI}}=3$, only $40 \%$ in scenario 1 and $30 \%$ in scenario 2 of the countrywide spectrum can be allocated to SBSs of MNO 1 on each floor. Hence, the $28 \mathrm{GHz}$ licensed spectrum and the $60 \mathrm{GHz}$ unlicensed spectrum allocated to SBSs of MNO 1 on each floor with $I_{\mathrm{CCI}}=0$ are 2.5 times $(1 / 0.4)$ and 3.33 times $(1 / 0.3)$, respectively, of the allocated spectrum $I_{\mathrm{CCI}}=3$.

Since the achievable capacity depends directly on the amount of available spectrum, the maximum and minimum average capacities, $\mathrm{SE}, \mathrm{EE}$, and $\mathrm{CE}$ for $\mathrm{MNO} 1$ can be achieved, respectively, with $I_{\mathrm{CCI}}=0$ and $I_{\mathrm{CCI}}=3$ in both scenarios as shown in Figure 5. Moreover, Figure 5 also shows that the proposed technique with no CCI provides 2.5 times higher in scenario 1 and 2.8 times higher in scenario 2 average capacity, SE, EE, and CE performances than that with the maximum CCI. Furthermore, with regard to the traditional SLSA, the proposed technique improves the average capacity, SE, EE, and CE of MNO 1 by 3 times, 1.65 times, $75 \%$, and $60 \%$, respectively, in scenario 1 , whereas by 6.12 times, 5.104 times, $85.8 \%$, and $83.15 \%$, respectively, in scenario 2 , with no co-channel interferer UEs, $I_{\mathrm{CCI}}=0$. The values of the above performance metrics indicate the importance of operating the existing and future networks in the unlicensed bands along with the licensed bands to enhance the network performance significantly (e.g., 2.04 times in average capacity, 3.09 times in SE, $14.4 \%$, in EE, and $38.58 \%$ in $\mathrm{CE}$ improvements when operating additionally in the $60 \mathrm{GHz}$ unlicensed band in scenario 2 with respect to operating only in the $28 \mathrm{GHz}$ licensed band in scenario 1). Moreover, the improvement factors, in general, decrease as 


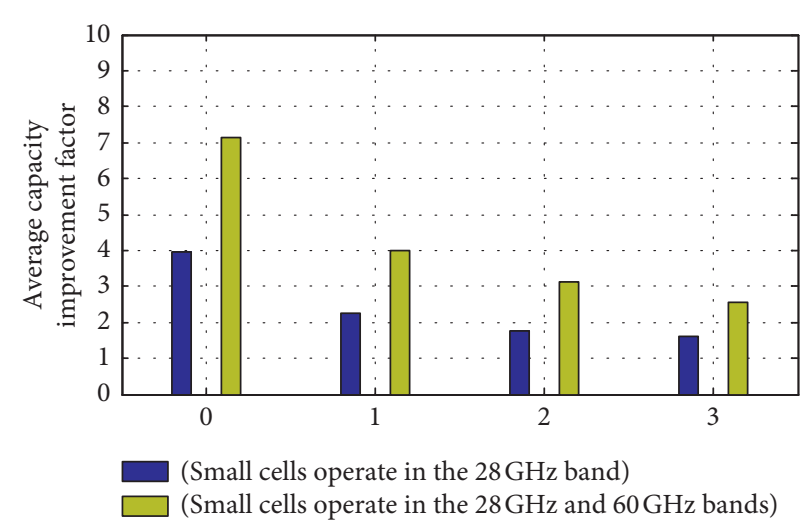

(a)

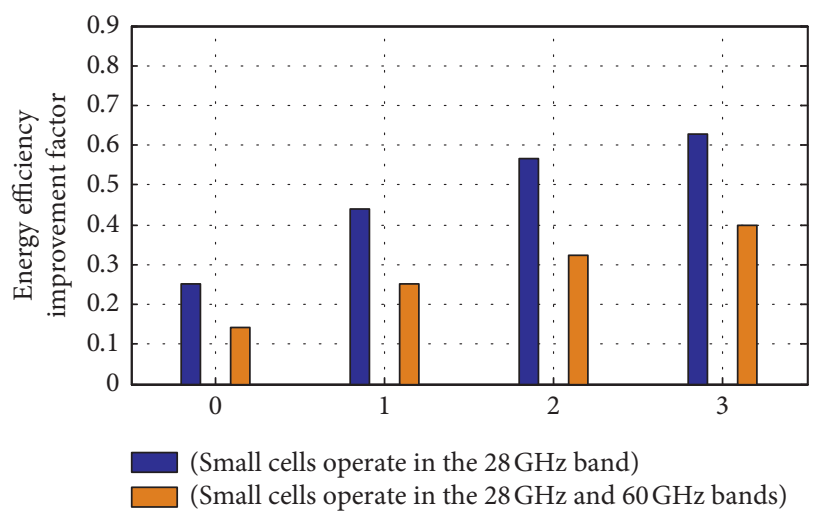

(c)

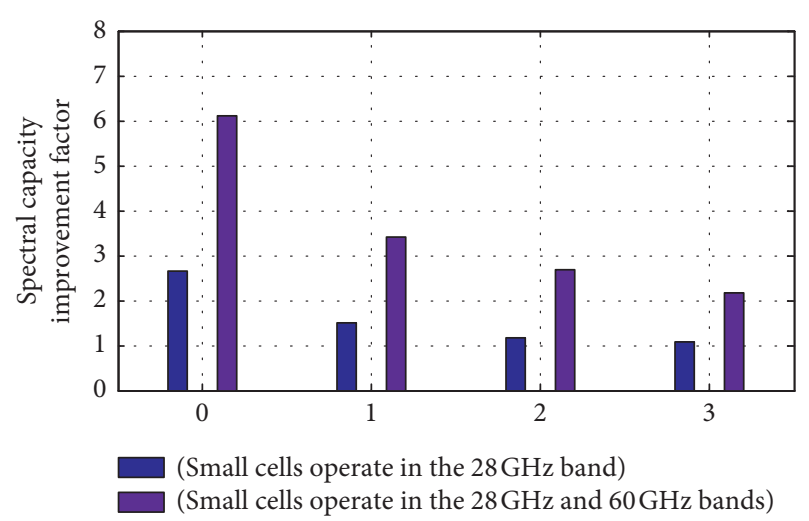

(b)

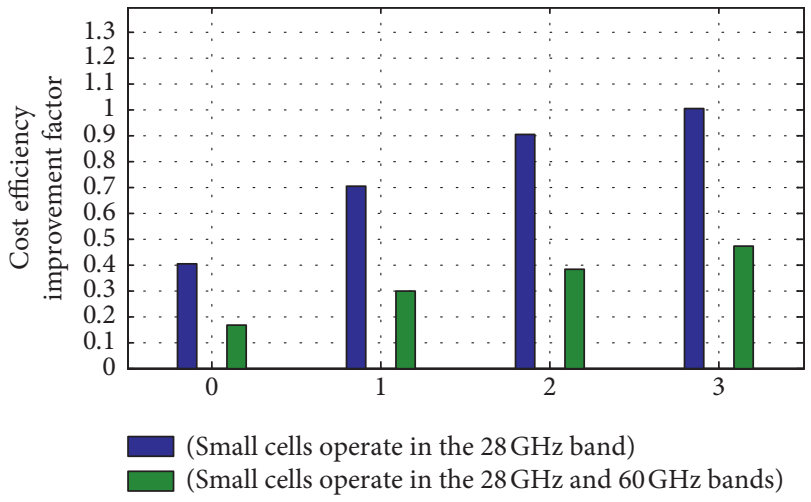

(d)

Figure 5: Performance improvement of the proposed technique (when small cells operate in the $28 \mathrm{GHz}$ spectrum, as well as when small cells operate in both the $28 \mathrm{GHz}$ and $60 \mathrm{GHz}$ bands) with respect to that of the traditional SLSA technique due to the change in the number of co-channel interferer UEs of MNOs $O \backslash o=1$ per apartment on a single floor of a building for UEs of MNO 1 of a country with $O=4$ MNOs and a single WiGig operator. (a) Average capacity, (b) spectral efficiency, (c) energy efficiency, and (d) cost efficiency.

$I_{\mathrm{CCI}}$ increases and get to a minimum when $I_{\mathrm{CCI}}=3$ in both scenarios, irrespective of the type and number of operating spectrum bands. Hence, CCI plays a vital role in the overall performances of an MNO when small cells are allocated to the countrywide either only licensed, or both licensed and unlicensed, mmWave spectrum bands.

5.2.2. Effect of the Spectrum Reuse. Figures 6(a) and 5(b) show the effect of reusing the same countrywide $28 \mathrm{GHz}$ and $60 \mathrm{GHz}$ spectra both vertically to each floor of SBSs of MNO 1 in a multistory building, as well as horizontally to each building of SBSs over a macrocell coverage of MNO 1. As can be seen from Figure 6, the proposed technique provides better SE and EE performances than the traditional SLSA technique, with the variation in either $\omega_{\mathrm{FL}}$, or $L$, or both, irrespective of the level of CCI in both scenarios. Like $L=1$, scenario 2 provides better SE and EE performances than scenario 1 due to contributing additional capacity from the $60 \mathrm{GHz}$ unlicensed mmWave spectrum to the $28 \mathrm{GHz}$ licensed spectrum. Moreover, with an increase in the number of floors $\omega_{\mathrm{FL}}$, i.e., vertical reuse factor (RF), as well as the number of buildings $L$, i.e., horizontal RF (hRF), SE increases linearly, whereas EE increases negative exponentially, irrespective of the degree of
CCI. Note, however, that since SE is affected additionally by the optimal amount of countrywide spectrum, the proposed technique in both scenarios with the maximum CCI provides insignificant SE while noticeable EE improvement over the traditional SLSA technique because of its higher average capacity performance, as shown in Figures 6(a) and 5(b).

Hence, the performance of the proposed technique in scenario 1 and scenario 2 is greatly influenced by the CCI, the operation in the unlicensed bands, and the spectrum RF. A significant performance gain can be achieved when the aggregate CCI is limited to a low value, small cells operate in the unlicensed mmWave bands in addition to the licensed mmWave bands, and spectrum RFs are increased noticeably. However, due to a small coverage of an indoor small cell in the high-frequency mmWave spectrum bands and a low probability of co-existing all interferer UEs of MNOs $\mathbf{O}$ $\backslash o=1$ within an apartment, the proposed technique can improve considerably the average SE and EE performances in scenarios 1 and 2. Moreover, the impact of CCI can be compensated by increasing the RF. For example, the proposed technique in scenario 1 with the maximum CCI for vertical RF $(\mathrm{vRF})=6$ can provide better SE and $\mathrm{EE}$ performances than when operating under no CCI scenarios for $\mathrm{vRF}=1$ for any hRF. 


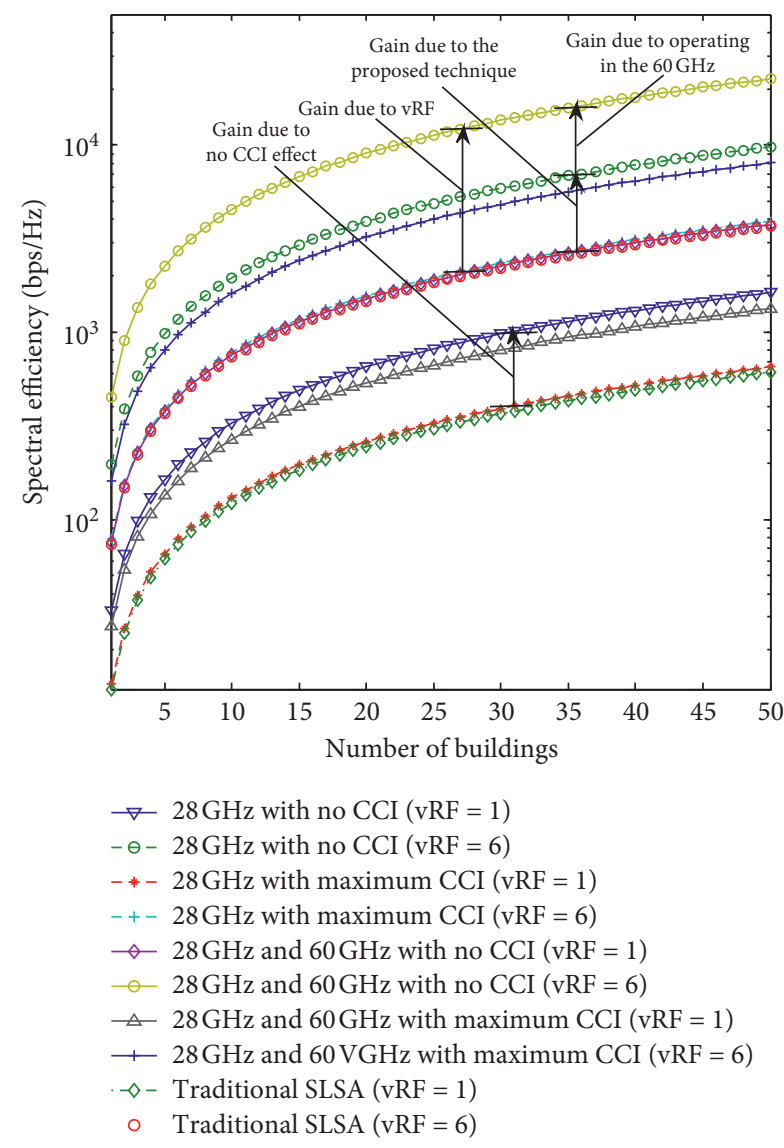

(a)

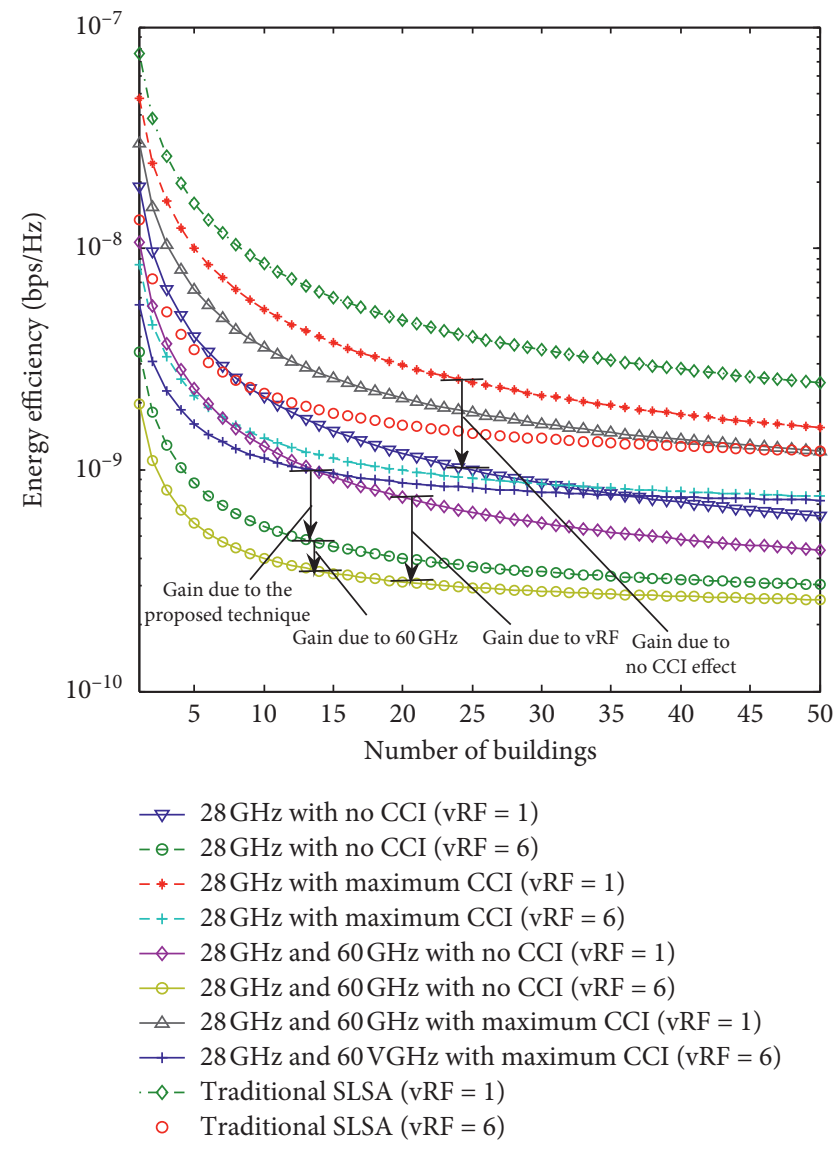

(b)

FIgURE 6: (a) SE and (b) EE performances of the proposed technique versus SLSA with a change in vRF and hRF for MNO 1.

TABLE 4: Required VRF and HRF for the proposed and SLSA techniques to satisfy SE and EE requirements for 6G mobile systems.

\begin{tabular}{|c|c|c|c|c|c|c|c|c|c|}
\hline \multirow{3}{*}{ Vertical RF } & \multicolumn{9}{|c|}{ Horizontal RF } \\
\hline & \multicolumn{2}{|c|}{$\begin{array}{c}\text { Proposed } \\
\text { technique } \\
\text { with no } \\
\text { CCI }\end{array}$} & \multicolumn{2}{|c|}{$\begin{array}{l}\text { Proposed } \\
\text { technique } \\
\text { with } \\
\text { maximum } \\
\quad \text { CCI }\end{array}$} & \multicolumn{2}{|c|}{$\begin{array}{l}\text { Traditional } \\
\text { SLSA }\end{array}$} & $\begin{array}{l}\text { Proposed technique } \\
\text { with no CCI }\end{array}$ & $\begin{array}{l}\text { Proposed technique } \\
\text { with maximum CCI }\end{array}$ & \multirow[t]{2}{*}{$\begin{array}{l}\text { Traditional } \\
\text { SLSA }\end{array}$} \\
\hline & SE & $\mathrm{EE}$ & SE & $\mathrm{EE}$ & SE & $\mathrm{EE}$ & \multicolumn{2}{|c|}{ Minimum required to satisfy $\mathrm{SE}$ and $\mathrm{EE}$} & \\
\hline \multicolumn{10}{|c|}{ Scenario 1 (when small cells operate only in the $28 \mathrm{GHz}$ licensed spectrum band) } \\
\hline 1 & 12 & 1 & 29 & 1 & 31 & 1 & 12 & 29 & 31 \\
\hline 6 & 2 & 1 & 5 & 1 & 6 & 1 & 2 & 5 & 6 \\
\hline \multicolumn{10}{|c|}{ Scenario 2 (when small cells operate in both the $28 \mathrm{GHz}$ licensed spectrum and the $60 \mathrm{GHz}$ unlicensed spectrum band) } \\
\hline 1 & 5 & 1 & 14 & 1 & 31 & 1 & 5 & 14 & 31 \\
\hline 6 & 1 & 1 & 3 & 1 & 6 & 1 & 1 & 3 & 6 \\
\hline
\end{tabular}

5.3. Performance Comparison. According to [36, 37], the future $6 \mathrm{G}$ mobile systems are expected to require 10 times average SE (i.e., $270-370 \mathrm{bps} / \mathrm{Hz}$ ) as well as 10 times average $\mathrm{EE}$ (i.e., $0.3 \times 10^{-6}$ Joules/bit) of that of $5 \mathrm{G}$ mobile systems $[38,39]$. Using Figure 6 , Table 4 shows the variation in the required values of the $\mathrm{vRF} \omega_{\mathrm{FL}}$ and hRF $L$ when employing the traditional SLSA technique and the proposed technique with no $\mathrm{CCI}$, as well as the maximum CCI, for each apartment on each floor of any building for MNO 1 in scenario 1 and scenario 2 to satisfy both the SE and EE requirements for $6 \mathrm{G}$ mobile systems.

From Table 4, it can be found that the required SE and EE for $6 \mathrm{G}$ can be achieved by changing either vRF $\omega_{\mathrm{FL}}$ or hRF $L$ such that their product, i.e., $\left(\omega_{\mathrm{FL}} \times L\right)$, defines the achievable $\mathrm{SE}$ and EE performances. Moreover, the proposed technique can satisfy both the SE of $370 \mathrm{bps} / \mathrm{Hz}$ and $\mathrm{EE}$ of $0.3 \mu \mathrm{J} / \mathrm{bit}$ for 
6G mobile systems by reusing (horizontally) the countrywide $28 \mathrm{GHz}$ mmWave spectrum to small cells of MNO 1 of about $61.2 \%$ less number of single-floor (i.e., $\omega_{\mathrm{FL}}=1$ ) buildings (i.e., $L=12$ ) with no CCI, whereas $6.4 \%$ less number of single-floor buildings (i.e., $L=29$ ) with the maximum CCI than that required by the traditional SLSA technique (i.e., $L=31$ ).

However, in scenario 2 , when small cells operate in both the $60 \mathrm{GHz}$ unlicensed spectrum and the $28 \mathrm{GHz}$ licensed spectrum, the same $\mathrm{SE}$ and $\mathrm{EE}$ requirements for $6 \mathrm{G}$ can be satisfied by reusing (horizontally) both $\mathrm{mmW}$ ave spectra to small cells of MNO 1 of about $83.87 \%$ less number of single-floor (i.e. $\omega_{\mathrm{FL}}=1$ ) buildings (i.e., $L=5$ ) with no CCI, whereas $54.83 \%$ less number of single-floor buildings (i.e., $L=14$ ) with the maximum CCI than that required by the traditional SLSA technique (i.e., $L=31$ ). Hence, this implies that scenario 2 (due to operating both in the $28 \mathrm{GHz}$ licensed and $60 \mathrm{GHz}$ unlicensed bands) outperforms scenario 1 (due to operating only in the $28 \mathrm{GHz}$ licensed band) by reusing the same spectrum to SBSs of $58.33 \%$ (i.e., $L=5$ ) less number of single-floor (i.e. $\omega_{\mathrm{FL}}=1$ ) buildings with no CCI, whereas $51.72 \%$ (i.e., $L=14$ ) with the maximum CCI than that required by scenario 1 by reusing the same spectrum to SBSs of $L=12$ number of single-floor buildings with no CCI and $L=29$ number of single-floor buildings with the maximum CCI.

\section{Offered Benefits and Further Outlooks}

6.1. Offered Benefits. The proposed technique benefits from a number of issues as follows. Unlike the traditional SLSA technique, the proposed technique ensures the availability of a large amount of spectrum by allocating the countrywide full (instead of a portion) mmWave spectrum to each MNO. Further, it provides an efficient spectrum utilization by allowing each MNO dynamic and flexible (instead of static and dedicated) access to the countrywide spectrum. Furthermore, it allows an MNO to pay only for the amount of spectrum that it uses to serve its user demands (i.e., in proportionate with the number of its users) at any term $t_{\text {rnw }}$, resulting in reducing the cost per unit capacity (i.e., bps).

\subsection{Further Outlooks}

6.2.1. Modeling 3D Spectrum Reuse. In this paper, we limit reusing the same countrywide full spectrum in small cells of an MNO $o$ on each floor (i.e., 2-dimensional space) of a multistory building. However, the countrywide full spectrum allocated to an MNO $o$ in the primary-level can be exploited in the 3D space of a multistory building of small cells to increase the vertical RF even further for a building. More specifically, by enforcing a maximum CCI, a minimum distance between co-channel small cells (each located in an apartment) can be defined in both the intrafloor and interfloor levels to form a 3D cluster of small cells of an MNO $o$ within a building. The allocated spectrum per MNO can then be reused to each $3 \mathrm{D}$ cluster of small cells of an
MNO $o$ to improve the spectrum utilization. For example, adopting [17], a minimum distance between co-channel small cells for the $28 \mathrm{GHz}$ mmWave spectrum in the intrafloor level and interfloor level, respectively, for any MNO $o$ at any term $t_{\text {rnw }}$ can be expressed as follows:

$$
\begin{aligned}
& \Delta_{a}=\Delta_{m} \times\left(\frac{\Xi_{a}}{I_{a}^{\mathrm{thr}}}\right)^{(1 / 1.797)} \\
& \Delta_{e} \geq \Delta_{m} \times\left(\frac{\left(\Xi_{e} / I_{e}^{\mathrm{thr}}\right)}{10\left(\alpha_{f}\left(\Delta_{e}\right) / 10\right)}\right)^{(1 / 1.797)}
\end{aligned}
$$

where $I_{a}^{\text {thr }}$ and $I_{e}^{\text {thr }}$ denote, respectively, intrafloor and interfloor CCI constraints at a small cell UE. $\Xi_{a}$ and $\Xi_{e}$ denote, respectively, the maximum number of co-channel small cells in the intrafloor level and interfloor level. $\Delta_{m}$ denotes the minimum distance between a co-channel small cell and a small cell UE and $\alpha_{f}\left(\Delta_{e}\right)$ denotes the floor penetration loss at the $28 \mathrm{GHz}$.

Let $s_{l}^{a}$ and $s_{l}^{e}$ denote, respectively, the number of small cells corresponding to $\Delta_{a, l}$ and $\Delta_{e, l}$ in a building $l$ such that a $3 \mathrm{D}$ cluster consists of $S_{3 \mathrm{D}, l}=\left(s_{l}^{a} \times s_{l}^{e}\right)$ small cells. Hence, the same spectrum of MNO $o$ can be reused for each cluster of $\left(s_{l}^{a} \times s_{l}^{e}\right)$ small cells in a building. Let $S_{F, l}$ denote the maximum number of small cells of an $\mathrm{MNO} o$ in a building $l$ such that the number of times the same spectrum of MNO $o$ can be reused in building $l$ (i.e., the spectrum RF for MNO $o$ inbuilding $l$ ) can be expressed as follows:

$$
\begin{aligned}
& \omega_{3 \mathrm{D}, l}=\frac{S_{F, l}}{\left(s_{l}^{a} \times s_{l}^{e}\right)}, \\
& \omega_{3 \mathrm{D}, l}=\frac{S_{F, l}}{S_{3 \mathrm{D}, l}} .
\end{aligned}
$$

Since the spectrum reuse can be performed to small cells deployed on the same floor of a building and the $28 \mathrm{GHz}$ mmWave signal faces high floor penetration loss, $\omega_{3 \mathrm{D}, l} \geq \omega_{\mathrm{FL}, l}$ may satisfy, resulting in improving the average capacity, SE, EE, and CE even further. We consider this issue as a part of our future studies.

6.2.2. Impact of Frequency Bands on Spectrum Exploitation. Figure 7 shows the path loss responses with the variation in carrier frequency bands between an SBS and its UE. The parameters and assumptions used for the path loss estimation are given in Table 5. It can be observed that the path loss is the most in the $140 \mathrm{GHz} \mathrm{THz}$ band and decreases with a corresponding decrease in the carrier frequency. This is because a high-frequency signal gets affected more by the propagating environment than that of a low-frequency one. Since the usable mmWave frequencies for $5 \mathrm{G}$ as well as the $\mathrm{THz}$ frequencies for the upcoming 6G range largely, there is a corresponding impact on the reuse of the spectrum. For example, the distance-dependent path loss for the $60 \mathrm{GHz}$ mmWave band can be expressed as [41]. 


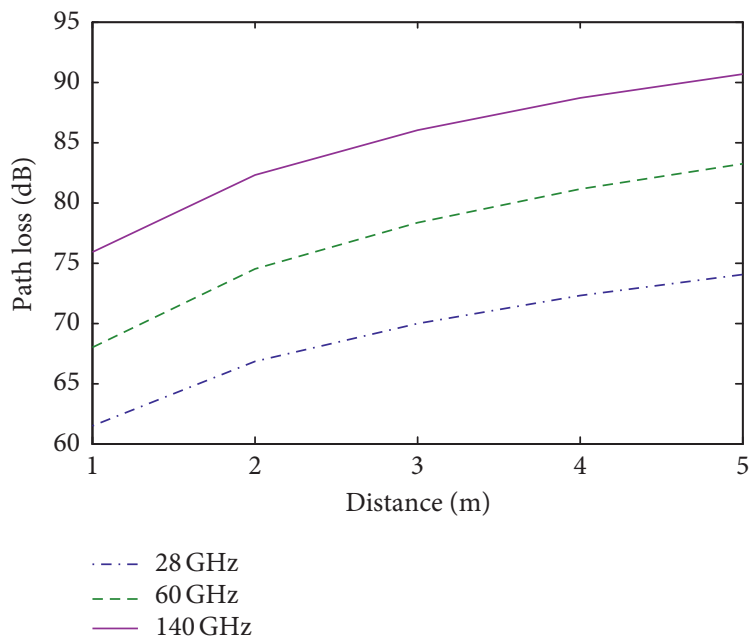

FIgURE 7: Path loss responses within a building between an SBS and its UE with a variation in the carrier frequency.

TABLE 5: Parameters and assumptions for path loss estimation.

\begin{tabular}{|c|c|}
\hline Parameters and assumptions & Value \\
\hline Transmit direction & Downlink \\
\hline SBS operating bandwidth (for each carrier frequency) & $50 \mathrm{MHz}$ \\
\hline Transmission power $(\mathrm{dBm})^{6}$ (for each carrier frequency) & 10 \\
\hline Path loss $(140 \mathrm{GHz})^{6}$ & $75.89+21.17 \log _{10}(d)$ \\
\hline Lognormal shadowing standard deviation $(\mathrm{dB})$ & $0.5712(\text { for } 140 \mathrm{GHz})^{6}$ \\
\hline Small-scale fading model & No small-scale fading effect for $140 \mathrm{GHz}^{6}$ \\
\hline SBS antenna gain $^{6}$ (for $140 \mathrm{GHz}$ ) & $21 \mathrm{~dB}$ \\
\hline SUE antenna gain ${ }^{6}$ and noise figure ${ }^{6}$ (for $140 \mathrm{GHz}$ ) & $21 \mathrm{~dB}$ and $10 \mathrm{~dB}$ \\
\hline
\end{tabular}

${ }^{6}$ Taken from [40].

$P L(\mathrm{~dB})=68+21.7 \log _{10}(d)$, where $d$ is in the meter. Like the $28 \mathrm{GHz}$ band, adopting [42], a minimum distance between co-channel small cells for the path loss of $60 \mathrm{GHz}$ mmWave spectrum as given above in the intrafloor level and interfloor level, respectively, for any MNO $o$ can be expressed as follows:

$$
\begin{aligned}
& \Delta_{a, 60}=\Delta_{m} \times\left(\frac{\Xi_{a}}{I_{a}^{\mathrm{thr}}}\right)^{(1 / 2.17)}, \\
& \Delta_{e, 60} \geq \Delta_{m} \times\left(\frac{\left(\Xi_{e} / I_{e}^{\mathrm{thr}}\right)}{10\left(\alpha_{f}\left(\Delta_{e, 60}\right) / 10\right)}\right)^{(1 / 2.17)},
\end{aligned}
$$

where $\Delta_{m}, \Xi_{a}$, and $I_{a}^{\mathrm{thr}}$ for the intrafloor level, as well as $\Xi_{e}$ and $I_{e}^{\text {thr }}$ for the interfloor level, are the same for both the 28$\mathrm{GHz}$ and $60-\mathrm{GHz} \mathrm{mm}$ wave bands. Now, taking the ratio of (43) to (40), we can find the following for the intrafloor level:

$$
\begin{aligned}
& \frac{\Delta_{a, 60}}{\Delta_{a, 28}}=\left(\frac{\Xi_{a}}{I_{a}^{\mathrm{thr}}}\right)^{(1 / 2.17)-(1 / 1.797)}, \\
& \frac{\Delta_{a, 60}}{\Delta_{a, 28}}=\left(\frac{\Xi_{a}}{I_{a}^{\mathrm{thr}}}\right)^{-0.373} .
\end{aligned}
$$

However, $0 \leq I_{a}^{\mathrm{thr}} \leq 1$ and $\Xi_{a}$ is a positive integer such that the following holds:

$$
\left(\frac{\Xi_{a}}{I_{a}^{\mathrm{thr}}}\right)^{-0.373}<1
$$

Hence,

$$
\Delta_{a, 60}<\Delta_{a, 28}
$$

This implies that the minimum distance in the intrafloor level decreases with an increase in frequency. Moreover, due to the higher frequency band, $\alpha_{f}\left(\Delta_{e, 60}\right) \geq \alpha_{f}\left(\Delta_{e, 28}\right)$. Hence, following the above procedure for the intra-floor level, it can be shown that the following holds for the interfloor level:

$$
\Delta_{e, 60}<\Delta_{e, 28} \text {. }
$$

Let $s_{l, 60}^{a}$ correspond to $\Delta_{a, 60}$ and $s_{l, 60}^{e}$ corresponds to $\Delta_{e, 60}$ such that a 3D cluster at the $60 \mathrm{GHz}$ band consists of $s_{3 \mathrm{D}, l, 60}=\left(s_{l, 60}^{a} \times s_{l, 60}^{e}\right)$ small cells. Then, from (47) and (48), we can find the following:

$$
\begin{gathered}
\left(s_{l, 60}^{a} \times s_{l, 60}^{e}\right)<\left(s_{l, 28}^{a} \times s_{l, 28}^{e}\right), \\
s_{3 \mathrm{D}, l, 60}<s_{3 \mathrm{D}, l, 28} .
\end{gathered}
$$

Hence, the 3D cluster size decreases with an increase in frequency, i.e., more reuse of the same amount of spectrum bandwidth at the $60 \mathrm{GHz}$ band can be made than that of the $28 \mathrm{GHz}$ band for the same number of apartments in a building. Since there are other mmWave bands considered 
effective for the existing $5 \mathrm{G}$ mobile systems (e.g., $26 \mathrm{GHz}$, $39 \mathrm{GHz}$, and $73 \mathrm{GHz}$ ) and $\mathrm{THz}$ bands (e.g., above $100 \mathrm{GHz}$ ) for the upcoming $6 \mathrm{G}$ mobile systems, a detailed understanding of how the mmWave and $\mathrm{THz}$ spectrum bands impact their reuse in in-building scenarios is necessary for 5G and upcoming 6G mobile systems, which we consider addressing as further studies.

6.2.3. $\mathrm{mm}$ Wave Spectrum Allocation and Reuse in Outdoor Environments. In this paper, we limit investigating the proposed countrywide mmWave spectrum allocation and reuse technique to indoor SBSs deployed in multistory buildings. However, the propagation characteristics of mmWave signals in outdoor environments differ greatly from those in indoor ones, particularly, rain and atmospheric absorption effect, cell coverage, shadowing effect from large buildings, outage probability, user density, speed, mobility, and handover management. All these aspects have a significant impact on the allocation and reuse of the mmWave spectrum outdoors. Hence, how to allocate the countrywide mmWave spectrum to each MNO in outdoor environments without causing CCI to each other and reuse the same mmWave spectrum for any MNO spatially need considerable research works. We aim to address this issue of the mmWave spectrum allocation and reuse in outdoor environments in our future research studies.

\section{Conclusion}

In this paper, by exploiting the frequency-domain, we have proposed a countrywide millimeter-wave (mmWave) spectrum allocation and reuse technique that allocates and reuses spatially the countrywide $28 \mathrm{GHz}$ licensed spectrum and $60 \mathrm{GHz}$ unlicensed spectrum to each Fifth-Generation (5G) New Radio (NR) Mobile Network Operator (MNO) of an arbitrary country to operate its small cells per floor in a building. An interference management scheme has been developed to avoid Co-Channel Interference (CCI) among small cells of 5G NR MNOs within each apartment on each floor. We have modeled user statistics per small cell and cochannel interferer statistics per apartment and formulated the optimal amount of the $28 \mathrm{GHz}$ licensed and $60 \mathrm{GHz}$ unlicensed mmWave spectra of each MNO. We have derived average capacity, spectral efficiency (SE), energy efficiency (EE), and cost efficiency (CE) of each MNO when employing the proposed technique, as well as the traditional Static Licensed Spectrum Allocation (SLSA) technique that allocates an equal amount of the $28 \mathrm{GHz}$ licensed spectrum to each MNO. We have shown analytically the outperformance of the proposed technique over the SLSA technique and discussed the implementation of the proposed technique by presenting a hybrid centralized-distributed coordination scheme.

The performance of the proposed technique has been evaluated under two scenarios, namely, small cells of each MNO operate only in the $28 \mathrm{GHz}$ licensed band in scenario 1 and both $28 \mathrm{GHz}$ licensed and $60 \mathrm{GHz}$ unlicensed bands in scenario 2, with regard to the traditional SLSA technique. By varying CCI and spectrum reuse factor, extensive numerical and simulation results and analyses have been carried out for a country consisting of four MNOs, i.e., MNOs 1, 2, 3, and 4, with a subscriber base of, respectively, 40\%, 30\%, 20\%, and $10 \%$ of the countrywide subscribers in scenario 1 . However, in scenario 2, in addition to MNOs 1, 2, 3, and 4, with a subscriber base of, respectively, $30 \%, 25 \%, 15 \%$, and $10 \%$, an incumbent Wireless Gigabit (WiGig) operator has been considered with a subscriber base of the remaining $20 \%$ of the countrywide subscribers.

It has been shown that the proposed technique with no CCI can achieve 2.5 times and 3.33 times higher average capacity, SE, EE, and CE in scenario 1 and scenario 2, respectively, than that with the maximum CCI. Further, with regard to the traditional SLSA, the proposed technique with no CCI can improve the average capacity, SE, EE, and CE of MNO 1 by 3 times, 1.65 times, 75\%, and 60\%, respectively, in scenario 1, whereas 6.12 times, 5.104 times, 85.8\%, and $83.15 \%$, respectively, in scenario 2 . Hence, when operating additionally in the $60 \mathrm{GHz}$ unlicensed band in scenario 2 with respect to operating only in the $28 \mathrm{GHz}$ licensed band in scenario 1, the network performance enhances significantly (i.e., 2.04 times in average capacity, 3.09 times in SE, 14.4\%, in $\mathrm{EE}$, and $38.58 \%$ in $\mathrm{CE}$ ), indicating the importance of operating the existing and future networks in the unlicensed mmWave bands along with the licensed mmWave bands. With an increase in the number of co-channel interferers $\mathrm{I}_{\mathrm{CCI}}$, the above improvement factors decrease and get to a minimum when $\mathrm{I}_{\mathrm{CCI}}$ is the maximum, i.e. 3 , in both scenarios.

Moreover, with an increase in the vertical reuse factor $(\mathrm{vRF}) \omega_{\mathrm{FL}}$ and horizontal reuse factor (hRF) $L$, SE increases linearly and EE increases negative exponentially, irrespective of the value of $\mathrm{I}_{\mathrm{CCI}}$. Overall, the performance gain improves with a decrease in CCI and an increase in vRF and hRF when small cells operate in scenario 2. Further, we have shown that the proposed technique can satisfy SE and EE requirements for sixth-generation (6G) mobile systems by reusing the spectrum in scenario 1 and scenario 2 , respectively, to small cells of MNO 1 of about $61.2 \%$ and $83.87 \%$ less number of buildings with no CCI, whereas $6.4 \%$ and $54.83 \%$ less number of buildings with the maximum CCI than that required by SLSA for $\mathrm{vRF}=1$. Finally, we have discussed offered benefits and point out key issues of the proposed technique for further studies.

\section{Data Availability}

Data, primarily, in the form of numerous simulation assumptions and parameters reported previously by the standardization bodies, including $3^{\text {rd }}$ Generation Partnership Project (3GPP) [31, 32] and International -Telecommunication Union-Radiocommunication Sector (ITU-R) [33], included and detailed within the article in Table 3, were used to carry out the performance evaluation of this study. Other prior studies than these above [31-33] were cited at relevant places within the text as references [34, 36-39, 42]. No data other than these were used to evaluate the performance studies. Taking into account all these parameters and assumptions, performance results were generated by a simulator running on a personal computer, which was built 
by the author using the standard computational tool MATLAB R2012b. MATLAB codes are not publicly available. However, supports for writing MATLAB instruction codes can be provided over the emails querying directly to the author at rony107976@gmail.com.

\section{Disclosure}

This paper was presented partly as a conference article that received the "Best Paper Award" at the Fifteenth International Conference on Systems and Networks Communications (ICSNC), Porto, Portugal, 2020 [23]. This research does not receive any funding.

\section{Conflicts of Interest}

The author declares that there are no conflicts of interest regarding the publication of this paper.

\section{References}

[1] S. Lagen, N. Patriciello, and L. Giupponi, "Cellular and wi-fi in unlicensed spectrum: competition leading to convergence," 2020 2nd 6G Wireless Summit (6G SUMMIT), vol. 2020, pp. 1-5, 2020.

[2] Y. Huang, Y. Chen, Y. T. Hou, W. Lou, and J. H. Reed, "Recent advances of LTE/WiFi coexistence in unlicensed spectrum," IEEE Network, vol. 32, no. 2, pp. 107-113, 2018.

[3] R. Zhang, M. Wang, L. X. Cai et al., "LTE-unlicensed: the future of spectrum aggregation for cellular networks," IEEE Wireless Communications, vol. 22, no. 3, pp. 150-159, 2015.

[4] R. K. Saha, "Approaches to improve millimeter-wave spectrum utilization using indoor small cells in multioperator environments toward 6G," IEEE Access, vol. 8, pp. 207643-207658, 2020.

[5] T. Nihtilä, "System performance of LTE and IEEE 802.11 coexisting on a shared frequency band," in Proceedings of the 2013 IEEE Wireless Communications and Networking Conference (WCNC), pp. 1038-1043, Shanghai, China, 2013.

[6] A. K. Bairagi, N. H. Tran, W. Saad, Z. Han, and C. S. Hong, "A game-theoretic approach for fair coexistence between LTE-U and wi-fi systems," IEEE Transactions on Vehicular Technology, vol. 68, no. 1, pp. 442-455, 2019.

[7] A. K. Bairagi, S. F. Abedin, N. H. Tran, D. Niyato, and C. S. Hong, "QoE-enabled unlicensed spectrum sharing in 5G: a game-theoretic approach," IEEE Access, vol. 6, pp. 5053850554, 2018.

[8] M. Ali, S. Qaisar, M. Naeem, W. Ejaz, and N. Kvedaraite, "LTE-U WiFi HetNets: enabling spectrum sharing for 5G/ beyond 5G systems," IEEE Internet of Things Magazine, vol. 3, no. 4, pp. 60-65, 2020.

[9] G. Naik, J.-M. Park, J. Ashdown, and W. Lehr, "Next generation wi-fi and 5G NR-U in the $6 \mathrm{GHz}$ bands: opportunities and challenges," IEEE Access, vol. 8, pp. 153027-153056, 2020.

[10] S. Lagen, L. Giupponi, S. Goyal et al., "New radio beam-based access to unlicensed spectrum: design challenges and solutions," IEEE Communications Surveys \& Tutorials, vol. 22, no. 1, pp. 8-37, 2020.

[11] Y. Jiang, J. Guo, and Z. Fei, "Performance analysis of the coexistence of 5G NR-unlicensed and wi-fi with mode selection," 2020.
[12] X. Lu, M. Lema, T. Mahmoodi, and M. Dohler, "Downlink data rate analysis of 5G-U (5G on unlicensed band): coexistence for 3GPP 5G and IEEE802.11ad WiGig," 2017.

[13] E. Almeida, "Enabling LTE/WiFi coexistence by LTE Blank subframe allocation," 2013.

[14] X. Yan, S. Qijun, Z. Hongshun, and S. Lulu, "Dynamic spectrum allocation based on cognitive radio," 2009.

[15] Z. Wei, D. Yang, and L. Sang, "Dynamic system level frequency spectrum allocation scheme based on cognitive radio technology," China Communications, vol. 11, no. 7, pp. 84-91, 2014.

[16] H. Shajaiah, A. Khawar, A. Abdel-Hadi, and T. C. Clancy, "Resource allocation with carrier aggregation in LTE advanced cellular system sharing spectrum with S-band radar," 2014.

[17] S. J. Kim, E. C. Kim, S. Park, and J. Y. Kim, "Dynamic spectrum allocation with variable bandwidth for cognitive radio systems," 2009.

[18] J. Gu, "Dynamic spectrum allocation algorithm for resolving channel conflict in cognitive vehicular networks," 2017.

[19] R. K. Saha and C. Aswakul, "A tractable Analytical model for interference characterization and minimum distance enforcement to reuse resources in three-dimensional in-building dense small cell networks," International Journal of Communication Systems, vol. 30, no. 11, p. e3240, 2017.

[20] R. K. Saha, "Modeling interference to reuse millimeter-wave spectrum to in-building small cells toward 6G," 2020.

[21] N. Saquib, E. Hossain, and D. Kim, "Fractional frequency reuse for interference management in LTE-advanced hetnets," IEEE Wireless Communications, vol. 20, no. 2, pp. 113-122, 2013.

[22] R. A. Hassan, A. Idris, H. Adto, M. Ramadhan, and M. Kassim, "Reduction of inter-cell interference in close proximity cell using dynamic fractional frequency reuse method," 2017.

[23] R. K. Saha, "A massive millimeter-wave spectrum allocation and exploitation technique toward 6G mobile networks," 2020.

[24] R. K. Saha, S. Nanba, and K. Nishimura, "A technique for cloud based clustering and spatial resource reuse and scheduling of 3D in-building small cells using CoMP for high capacity CRAN," IEEE Access, vol. 6, pp. 71602-71621, 2018.

[25] H. A. U. Mustafa, M. A. Imran, M. Z. Shakir, A. Imran, and R. Tafazolli, "Separation framework: an enabler for cooperative and D2D communication for future $5 \mathrm{G}$ networks," IEEE Communications Surveys \& Tutorials, vol. 18, no. 1, pp. 419-445, 2016.

[26] Q. Cui, H. Song, H. Wang, M. Valkama, and A. A. Dowhuszko, "Capacity analysis of joint transmission comp with adaptive modulation," IEEE Transactions on Vehicular Technology, vol. 66, no. 2, pp. 1876-1881, 2017.

[27] J. D. Chimeh, M. Hakkak, and S. A. Alavian, "Internet traffic and capacity evaluation in UMTS downlink," Future Generation Communication and Networking, vol. 2007, 2007.

[28] L. Kleinrock, Queueing Systems: Theory, Wiley, Hoboken, NJ, USA, 1975.

[29] R. K. Saha and C. Aswakul, "A novel frequency reuse technique for in-building small cells in dense heterogeneous networks," IEEJ Transactions on Electrical and Electronic Engineering, vol. 13, no. 1, pp. 98-111, 2018.

[30] R. H. Tehrani, S. Vahid, D. Triantafyllopoulou, H. Lee, and K. Moessner, "Licensed spectrum sharing schemes for mobile operators: a survey and outlook," IEEE Communications Surveys \& Tutorials, vol. 18, no. 4, pp. 2591-2623, 2016. 
[31] Evolved Universal Terrestrial Radio Access (E-UTRA); Radio Frequency (RF) System Scenarios, "Document 3GPP TR 36.942, V.1.2.0, 3rd generation partnership project," 2007.

[32] Simulation Assumptions and Parameters for FDD HeNB RF Requirements, "Document TSG RAN WG4 (radio) meeting \#51, R4-092042, 3GPP,” 2009.

[33] Guidelines for Evaluation of Radio Interface Technologies for IMT-2020, “Report ITU-R M.2412-0 (10/2017)," 2017.

[34] G. R. Maccartney, T. S. Rappaport, S. Sun, and S. Deng, "Indoor office wideband millimeter-wave propagation measurements and channel models at 28 and $73 \mathrm{GHz}$ for ultra-dense 5G wireless networks," IEEE Access, vol. 3, pp. 2388-2424, 2015.

[35] S. Geng, J. Kivinen, X. Zhao, and P. Vainikainen, "Millimeterwave propagation channel characterization for short-range wireless Communications," IEEE Access, vol. 58, no. 1, pp. 3-13, 2009.

[36] Z. Zhang, Y. Xiao, Z. Ma et al., "6G wireless networks: vision, requirements, architecture, and key technologies," IEEE Vehicular Technology Magazine, vol. 14, no. 3, pp. 28-41, 2019.

[37] S. Chen, Y.-C. Liang, S. Sun, S. Kang, W. Cheng, and M. Peng, "Vision, requirements, and technology trend of 6G: how to tackle the challenges of system coverage, capacity, user datarate and movement speed," IEEE Wireless Communications, vol. 27, no. 2, pp. 218-228, 2020.

[38] C.-X. Wang, F. Haider, X. Gao et al., "Cellular architecture and key technologies for 5G wireless communication networks," IEEE Communications Magazine, vol. 52, no. 2, pp. 122-130, 2014.

[39] G. Auer, V. Giannini, C. Desset et al., "How much energy is needed to run a wireless network?" IEEE Wireless Communications, vol. 18, no. 5, pp. 40-49, 2011.

[40] N. A. Abbasi, A. Hariharan, A. M. Nair, and A. F. Molisch, "Channel measurements and path loss modeling for indoor THz communication," 2020.

[41] R. K. Saha and C. Aswakul, "Incentive and architecture of multi-band enabled small cell and UE for up-/down-link and control-/user-plane splitting for 5G mobile networks," Frequenz, vol. 71, no. 1-2, pp. 95-118, 2017.

[42] R. K. Saha, "3D spatial reuse of multi-millimeter-wave spectra by ultra-dense in-building small cells for spectral and energy efficiencies of future 6G mobile networks," Energies, vol. 13, no. $7,2020$. 